\title{
On the relation between two conjectures on polynomials
}

A. Sornomzac (Warszawa)

x. The aim of this paper is to establish a relation between the conjecture $\mathrm{H}$ on simultaneous representation of primes by several irreducible polynomials (see [12] and [5]) and a conjecture on Diophantine equations with parameters that wo shall denote by 0 . Both conjectures involve the notion of the fixed divisor of a polynomial, i.e. the greatest common divisor of all values the polynomial takes for integral values of the argumonts. The conjectures run as follows.

H. Let $f_{1}(x), \ldots, f_{k}(x)$ be irreducible polynomials with integral coeffioients and the leading coefficients positive such that $\prod_{j=1}^{k} f_{j}(x)$ has the fixed divisor 1. Then there exist infinitely many positive integers $x$ such that all numbers $f_{j}(x)$ are primes.

c. Let $f^{\prime}(x, y) \in \boldsymbol{Z}[x, y]$ be a form such that

(1) $\quad F(x, y)=F_{1}(a x+b y, c x+d y)$ for any. $F_{1} \in \mathbf{Z}[x, y]$ and any

$$
a, b, e, d \in \boldsymbol{Z} \text { implies }\left|\begin{array}{ll}
a & b \\
c & d
\end{array}\right|= \pm 1 \text {. }
$$

If $f\left(t_{1}, \ldots, t_{r}\right) \in Z\left[t_{1}, \ldots, t_{r}\right]$ has the fixed divisor equal to its content and the equation

$$
H^{\prime}(x, y)=f\left(t_{1}, \ldots, t_{r}\right)
$$

is soluble in integers an, $y$ for all integral vectors $\left[t_{1}, \ldots, t_{r}\right]$ then there exist polynomials $X, Y \in \mathbb{Z}\left[t_{1}, \ldots, t_{r}\right]$ such that identically

$$
F\left(X\left(t_{1}, \ldots, t_{r}\right), X\left(t_{1}, \ldots, t_{r}\right)\right)=f\left(t_{1}, \ldots, t_{r}\right) .
$$

A conjecture simitar to 0 has been proposed by Chowla [3]. He has made no assumption (1) but required $F$ and $f$ to be irreducible and have the fixed divisor 1. The following example shows that this is not enough:

$$
F(x, y)=x^{2}+3 y^{2}, \quad f\left(t_{1}, t_{2}\right)=t_{1}^{2}+t_{1} t_{2}+t_{2}^{2} .
$$

In this example tho set of values of $F^{\prime}(x, y)$ and of $f\left(t_{1} ; t_{2}\right)$ is the same, but $F$ and $f$ are not equivalent by unimodular transformation, which 
answers in the negative a question of Chowla (ibid., p. 73) repeated in [9]. The condition imposed in $O$ on the fixed divisor of $f$ is essential, as the following example shows

$$
F(x, y)=2 x^{2} y^{3}, \quad f(t)=t^{3}(t+1)^{4} .
$$

Fere the solutions of the equations (2) are given by

$$
\begin{array}{lll}
x=2(t+1)^{2}, y=\frac{1}{2} t & \text { if } & t=0 \bmod 2, \\
x=\frac{1}{4}(t+1)^{2}, y=2 t & \text { if } & t=1 \bmod 2,
\end{array}
$$

but there are no integer-valued polynomials $X(t) ; \quad Y(t)$ satistying (3). Another example with $F$ primitive is given at the end of Section 2 .

One special case of $\mathrm{C}$ corresponding to $F=x^{2}+y^{2}$ has boen proved in [3] and [4]. Chowla has also indicated how his conjecture for $F^{\prime}(x, y)$ quadratic should follow from the special case $z=1$ of H. We shall extend these results in the following two theorems.

THEOREm 1. 0 holds if $F^{\prime}(x, y)=x^{\prime k} y^{l}(k \geqslant 1, l \geqslant 1)$ or if $W^{\prime}$ is quadratic and equivalent (properly or improperly) to every. form in its genus. For such and for no other quadratic $\mathbb{F} \mathrm{C}$ extends to all polynomials $f \in \boldsymbol{Z}\left[t_{1}, \ldots, t_{r}\right]$. form.

THEOREM 2. $\mathrm{H}$ implies $\mathrm{O}$ if $F$ is a quadratio form or a reducible oubic

We shall see (Corollary to Lemma 3 ) that $O$ implies the following, less precise but more general assertion.

D. Let $F(x, y) \in \boldsymbol{Z}[x, y]$ be any form and $f \in \boldsymbol{Z}\left[t_{1}, \ldots, t_{r}\right]$ any polynomial. If the equation (2) is soluble in integers $x, y$ for all integral vectors $\left[t_{1}, \ldots, t_{r}\right]$ then there exist polynomials $X, Y \in Q Q\left[t_{1}, \ldots, t_{r}\right]$ satisfying (3).

$\mathrm{D}$ has been proved for $F=x^{n}$ and any $r$ in [7] and [11] also for any irreducible quadratic $F$ and $r=1$ in [4], $r>1$ in [1.]; for reducible quadratic $F$ it follows easily. We shall show

THEOREM 3. Himplies D if F factorizes into two relatively prime factors in an imaginary quadratic field.

In virtue of Theorem $3 \mathrm{H}$ implies $\mathrm{D}$ for $I^{\prime}=x^{n}+y^{n}$. By a modification of the proof of that theorem in this special case we shall show yet

Treomery 4. El implies $\mathrm{C}$ if $F(x, y)=x^{n}+y^{n}(n \geq 2)$. Wr $n=2$ and for no other $n$ in question $\mathrm{C}$ extends to all polynomials $f \in \mathbf{Z}\left[t_{1}, \ldots, t_{4}\right]$.

At the cost of considerable technical complications indicated bricfly later one can extend Theorem 2 to all forms $T^{3}$ splitting completely over a cyclic field except those with all zoros conjugate and ron. The quantitative version of H formulated by Bateman and Iforn [1] (sce also [5]) implies $\mathrm{O}$ in the exceptional case at least for $r=1$. Similarly Theorem 3 can be extended to all forms $\not F$ that factorize into two distinct complex conjugate factors over an imaginary cyclic field.
2. In the sequel we shall use the vector notation and write $t$ instead of $\left[t_{1}, \ldots, t_{r}\right], \boldsymbol{t}^{\prime}$ instedad of $\left[t_{2}, \ldots, t_{r}\right],\|\boldsymbol{t}\|$ for $\max \left|t_{\boldsymbol{i}}\right|$. We shall denote the contient of a polynomial $f$ by $O(f)$, its total degree by $|f|$ and call a form $T^{\prime}$ satisfying (1) primary. The letters $\boldsymbol{N}, \boldsymbol{Z}, \boldsymbol{Q}$ denote the set of positive integers, the ring of integers and the rational field, respectively. For a fixed field $\boldsymbol{K} N$ denotes the norm from $\boldsymbol{K}$ to $\boldsymbol{Q}$ or from $\boldsymbol{K}(\boldsymbol{t})$ to $\boldsymbol{Q}(\boldsymbol{t})$. The entent of a polynomial orer $\boldsymbol{K}$ is an ideal of $\boldsymbol{K}$ but if $\boldsymbol{K}=\boldsymbol{Q}$ it is often identified with the positive generator of this ideal.

Lamma 1. Let $P \in \mathbb{Z}[t], p$ be a prime dividing neither the leading coefficient nor the aisoriminant of $P$. If $t_{0} \in Z, P\left(t_{0}\right) \equiv 0 \bmod p$ then either $P\left(t_{0}\right) \not 20 \bmod p^{2}$ or $P\left(t_{0}+p\right) \not \equiv 0 \bmod p^{2}$.

Proof. Donoting the leading coefficient of $P$ by $a$, the discriminant of $P$ by $D$ and its derivative by $P^{\prime}$ we have

$$
P(t) U(t)+P^{\prime}(t) V(t)=a D,
$$

whero $U, V \in Z[t]$. Setting $t=t_{0}$ we infer from $P\left(t_{0}\right) \equiv 0 \bmod p, a D$ $\neq 0 \bmod p$ that $P^{\prime}\left(t_{0}\right) \not 0 \bmod p$. Now from the expansion

$$
P\left(t_{0}+p\right)=P\left(t_{0}\right)+P^{\prime}\left(t_{0}\right) p+\frac{P^{\prime \prime}\left(t_{0}\right)}{2} p^{2}+\cdots
$$

we get $P\left(t_{0}+p\right)-P\left(t_{0}\right) \neq 0 \bmod p^{2}$, whence the assertion.

IJWMMA 2. If a quadratio form $t^{\prime}$ is primary then

$$
H^{\prime}=A G(x, y), \text { where } A \in \mathbb{Z}, G(x, y) \in \boldsymbol{Z}[x, y] \text {, }
$$

$A$ is square-free, the discriminant $\Delta$ of $G$ is either 1 or fundamental and $\left(\frac{\Delta}{p}\right)$ $=-1$ for every prime faotor $p$ of $A$.

Proof. If $G$ is reducible, $G=(a x+b y)\left(a^{\prime} x+b^{\prime} y\right)$ we have

and by (l)

$$
F(x, y)=(A a x+A b y)\left(a^{\prime} x+b^{\prime} y\right)
$$

$$
A\left|\begin{array}{cc}
a & b \\
a^{\prime} & b^{\prime}
\end{array}\right|= \pm 1, \quad A= \pm 1 \text { and } \Delta=\left|\begin{array}{ll}
a & b \\
a^{\prime} & b^{\prime}
\end{array}\right|^{2}=1 \text {. }
$$

If $a^{y}$ is irreduciblo, lot $\theta=a x^{2}+b x y+o y^{2}$, and let $\omega_{1}, \omega_{2}$ be a basis of tho ideal a $a\left(a, \frac{b+\sqrt{a}}{2}\right)$. Then wo huve for suitable integers $a_{1}, a_{2}, b_{1}, b_{2}$

$$
\begin{gathered}
a=a_{1} \omega_{1}+a_{2} \omega_{2}, \\
\frac{b+\sqrt{\Delta}}{2}=b_{1} \omega_{2}+b_{2} \omega_{2} .
\end{gathered}
$$

Let $K=Q(\sqrt{A})$ and lot us set

$$
F_{y}(x, y)=A a^{-1} N\left(x \omega_{1}+y \omega_{2}\right)
$$


Since $N \mathfrak{a}=|a|$ and $\left(\omega_{1}, \omega_{2}\right) \equiv 0 \bmod \mathfrak{a}$ we have

On the other hand

$$
F_{1}(x, y) \in \boldsymbol{Z}[x, y]
$$

$$
a x+\frac{b+\sqrt{\Delta}}{2} y=\left(a_{1} x+b_{1} y\right) \omega_{1}+\left(a_{2} x+b_{2} y\right) \omega_{2},
$$

hence

and by (1)

$$
F^{\prime}(x, y)=F_{1}\left(a_{1} x+b_{1} y, a_{2} x+b_{2} y\right)
$$

$$
\left|\begin{array}{ll}
a_{1} & b_{1} \\
a_{2} & b_{2}
\end{array}\right|= \pm 1
$$

It follows that $\left[a, \frac{b+\sqrt{\Delta}}{2}\right]$ is itself a basis for $a$ and by a well known result

$$
|a|=\frac{1}{\sqrt{|a|}} \mathrm{abs}\left|\begin{array}{cc}
a & \frac{b+\sqrt{\Delta}}{2} \\
a & \frac{b-\sqrt{\Delta}}{2}
\end{array}\right|
$$

where $d$ is the discriminant of $K$. It follows that $\Delta=d$ is a fundamental discriminant. If $A$ is not square-free or for some $p \mid A$ we have $\left(\frac{\Delta}{p}\right)=0$ or 1 then for a suitable prime ideal $p: N p \mid A$

Let pa have an integral basis $\left[\Omega_{1}, \Omega_{2}\right]$ and let us set

$$
F_{1}(x, y)=A a^{-1} N p_{p}^{-2} N\left(x \Omega_{1}+y \Omega_{2}\right)
$$

Since $N\left(\Omega_{1}, \Omega_{2}\right)=|a| N$ p we have

On the other hand

$$
H_{1}(x, y) \in \boldsymbol{Z}[x, y] \text {. }
$$

$$
\omega_{i} N p=o_{i} \Omega_{1}+d_{i} \Omega_{2} \quad(i=1,2)
$$

for suitable $c_{i}, d_{i} \in \boldsymbol{Z}$, hence

and we get

Now by (1)

$$
\left(\omega_{1} x+\omega_{2} y\right) N p=\left(a_{1} x+a_{2} y\right) \Omega_{1}+\left(d_{1} x+d_{2} y\right) \Omega_{2}
$$

$$
F(x, y)=P_{1}\left(c_{1} x+c_{2} y, d_{1} x+d_{2} y\right)
$$

$$
\left|\begin{array}{ll}
c_{1} & c_{2} \\
d_{1} & d_{2}
\end{array}\right|= \pm 1
$$

hence $\left[\omega_{1} N \mathfrak{p}, \omega_{\mathfrak{2}} N \mathfrak{p}\right]$ is a basis for ap and $\mathfrak{a} N \mathfrak{p}=\mathfrak{a p}$, a contradiction.
Remarlk. Similanly one can show that if a primary form $F(x, y)$ is irroduciblo and $F^{\prime}(\vartheta, 1)=0$ then $[1, \vartheta]$ can be extended to a basis of the ideal $(1,9)$.

Proof of Theorom 1. Consider first $F(x, y)=x^{k} y^{l}$ and let

$$
f(\boldsymbol{t})=c \prod_{v=1}^{n} f_{\nu}(\boldsymbol{t})^{e_{\nu}}
$$

bo the canonical fuctorization of $f$ into primitive irreducible polynomials with integral coofficionts. In view of the condition on the fixed divisor of $f$ for overy prime factor $p$ of $c$ there exists a vector $\boldsymbol{t}_{p} \in Z^{r}$ such that

$$
\prod_{\nu=1}^{n} f_{y}\left(\boldsymbol{t}_{p}\right)^{e_{y}} \neq 0 \bmod p
$$

It follows from (2) with $t=t_{p}$ that

$$
\operatorname{ord}_{p} e=k \alpha+l \beta
$$

whero $a=\operatorname{ord}_{p} x, \beta=\operatorname{ord}_{p} y$ and we get

$$
0= \pm \xi^{k} \eta^{l}, \quad \xi, \eta \in \boldsymbol{Z} .
$$

On the other hand we can assume that $f(\boldsymbol{t})$ depends upon $t_{1}$. Let $a_{0}\left(\boldsymbol{t}^{\prime}\right)$, $D\left(\boldsymbol{t}^{\prime}\right)$ be the leading coefficient and the discriminant respectively of $\prod_{v=1}^{n} f_{v}(t)$ with respect to $t_{1}$. Wo have $a_{0} D \neq 0$ and there exists a vector $\boldsymbol{t}_{0}^{y=1} \in Z^{r-1}$ such that

$$
a_{0}\left(\boldsymbol{t}_{0}^{\prime}\right) D\left(\boldsymbol{t}_{0}^{\prime}\right) \neq 0
$$

For every $\nu \leqslant n$ there exists a prime $p$ and an integer $t_{0}$ such that

Put

$$
f_{n}\left(t_{0}, \boldsymbol{t}_{0}^{\prime}\right) \equiv 0 \bmod p, \quad c a_{0}\left(\boldsymbol{t}_{0}^{\prime}\right) D\left(\boldsymbol{t}_{0}^{\prime}\right) \neq \equiv \bmod p .
$$

$$
P(t)=\prod_{p=1}^{n} f_{n}\left(t, \boldsymbol{t}_{0}^{\prime}\right)
$$

Since $a_{0}\left(\boldsymbol{t}_{0}^{\prime}\right) \neq 0$, the discriminant of $P(t)$ equals $D\left(\boldsymbol{t}_{0}^{\prime}\right)$. Hence by $(6)$ and Lomma $t$ there oxists a $t_{1} \in Z$ such that

$$
P\left(t_{1}\right)=0 \bmod p, \quad P\left(t_{1}\right) \not \equiv \bmod p^{2} .
$$

We infer from (4), (5) and (6) that

(8) $f_{v}\left(t_{1}, \boldsymbol{t}_{0}^{\prime}\right)=0 \bmod p, f_{v}\left(t_{1}, \boldsymbol{t}_{0}^{\prime}\right) \not \equiv 0 \bmod p^{2}, f_{\mu}\left(t_{1}, \boldsymbol{t}_{0}^{\prime}\right) \not \equiv 0 \bmod p$

$$
(\mu \neq \nu) \text {. }
$$

It follows from (2) with $t=\left[t_{1}, \boldsymbol{t}_{0}^{\prime}\right],(6)$ and $(8)$ that

$$
e_{p}=7 a \alpha_{p}+l \beta_{p} \text {, }
$$


where $\alpha_{\nu}=\operatorname{ord}_{p} x, \beta_{n}=\operatorname{ord}_{j} y$. Take now

$$
X_{0}(\boldsymbol{t})=\xi \prod_{v=1}^{n} f_{v}(\boldsymbol{t})^{\alpha_{v}}, \quad Y_{0}(\boldsymbol{t})=\eta \prod_{y=1}^{n} f_{v}(\boldsymbol{t})^{\mu^{\prime}}
$$

It follows from $(5)$ and $(9)$ that

$$
X_{0}(\boldsymbol{t})^{l_{i}} Y_{0}(\boldsymbol{t})^{l}= \pm f(\boldsymbol{t})
$$

If the sign on the right-hand side is positive we take $X=X_{0}, Y=X_{0}$. If the sign is negative and either $k$ or $z$ is odd, we talio $X \ldots \pm X_{0}$, $Y=+Y_{0}$. If the sign is negative and $\%, l$ are both even we get a contiadiction. Indeed by $(5) 0<0$, by $(9) e_{1}=0 \bmod 2$, henee by $(4) f(t) \leqslant 0$. Taking $\boldsymbol{t} \in \boldsymbol{Z}^{r}$ such that $f(\boldsymbol{t}) \neq 0$ we get from (2) $x^{k} y^{l}<0$, which is impossible.

Consider now the case of $F$ quadratic. By Lemma $2 F$ is of the form $A G(x, y)$, where $A$ is square-free, $G(x, y)$ is a primitive form with discriminant $\Delta,\left(\frac{\Delta}{p}\right)=-1$ for every prime factor $p$ of $A$ and either $\Delta=1$ or $\Delta$ is fundamental. In the first case $F(x, y)$ is equivalenti to an and for the latter form one can take $X(t)=f(t), Y(t)=1$. In the socond case if $G(\vartheta, 1)=0, \boldsymbol{K}=\boldsymbol{Q}(\vartheta)$ and $a$ is the ideal $(1, \vartheta)$ we have

$$
G(x, y)=\frac{N(x-\vartheta y)}{N \mathfrak{a}}
$$

Changing if necessary the sign of $A$ we can assume that

$$
F^{\prime}(x, y)=\frac{A}{N \mathfrak{a}} N(x-\vartheta y) .
$$

The solubility of the equation $N(\omega)=\frac{N \mathfrak{a}}{A} f(t)$ for ant $t \in Z^{r}$ implios by Theorem 1 of [14] the existence of a polynomial $\omega(\boldsymbol{t}) \in \boldsymbol{K}[\boldsymbol{t}]$ such that

$$
N(\omega(\boldsymbol{t}))=\frac{N_{\mathfrak{a}}}{A} f(\boldsymbol{t}) .
$$

Let $\mathfrak{b}=\sigma(\omega)$ and let

$$
\mathrm{ba}^{-1}=\prod_{i=1}^{j} \mathfrak{p}_{i}^{a_{i}} \prod_{i=1}^{j} \mathfrak{p}_{i}^{\prime \prime i} \prod_{i=1}^{k} q_{i}^{i}
$$

be the factorization of $\mathfrak{b a}^{-1}$ in prime ideals of $\boldsymbol{K}$. Fere $p_{i}$ are distinet pairwise non conjugate prime ideals of degree 1 in $\boldsymbol{K}, \mathfrak{p}_{i}^{\prime}$ is conjugato to $p_{i}$ and $q_{i}$ are prime ideals of degree 2 in $\boldsymbol{K}$. Since $A N\left(\mathrm{ba}^{-1}\right) \in \boldsymbol{Z}$ and $A$ has only prime ideal factors of degree 2 in $\boldsymbol{K}$ we get

hence

$$
\begin{aligned}
a_{i}+b_{i} \geqslant 0 & (1 \leqslant i \leqslant j), \\
2 c_{i}+1 \geqslant 0 & (1 \leqslant i \leqslant \text { h) },
\end{aligned}
$$

(12) $\max \left\{0, a_{i}\right\}+\min \left\{0, b_{i}\right\} \geqslant 0, \quad \max \left\{0, b_{i}\right\}+\min \left\{0, a_{i}\right\} \geqslant 0$

Let us consider the ideal

$$
a_{i} \geq 0 \quad(1 \leqslant i \leqslant k)
$$

$(1 \leqslant i \leqslant j)$,

$$
\mathfrak{c}=\prod_{i=1}^{j} \mathfrak{p}_{i}^{\min \left(0, b_{i}\right)-\min \left(0, a_{i}\right)} \mathfrak{p}_{i}^{\prime \min \left(0, a_{i}\right)-\min \left(0, b_{i}\right)}
$$

Since $F$ is equivalent to every form in its genus the same is true about $G$, thus there is only one narrow class in the genus of a or there are two such classes represented by $\mathfrak{a}$ and $\mathfrak{a}^{\prime}$. In any case the principal genus consists only of the principal class and the class of $\mathfrak{a}^{2}$. Since $p_{i}^{\prime} \sim \mathfrak{p}_{i}^{-1}, c$ belongs to the principal genus and we get $c \sim 1$ or $c \sim \mathfrak{a}^{2}$. In the former case let $c=\left(\gamma_{1}\right)$ with $\gamma_{x}$ totally positive and consider the polynomial

\section{We have}

$$
\omega_{1}(\boldsymbol{t})=\gamma_{1} \omega(\boldsymbol{t})
$$

$$
C\left(\omega_{1}\right)=\left(\gamma_{1}\right) O(\omega)=\mathfrak{c b}=\mathfrak{a} \prod_{i=1}^{j} \mathfrak{p}_{i}^{\max \left\{0, a_{i}\right\}+\min \left\{0, b_{i}\right\}} \prod_{i=1}^{j} \mathfrak{p}_{i}^{\prime \max \left\{0, b_{i}\right\}+\min \left\{0, a_{i}\right\}} \prod_{i=1}^{k} q_{i}^{e}{ }^{i}
$$

and by $(12) O\left(\omega_{1}\right) \equiv 0 \bmod a$.

It follows that all the coefficients of $\omega_{1}$ are in $a$ and since by Lemma 2 $[1, \vartheta]$ is a basis of $\mathfrak{a}$ we get

$$
\omega_{1}(\boldsymbol{t})=X_{1}(\boldsymbol{t})-\vartheta Y_{1}(\boldsymbol{t}),
$$

where $X_{1}, Y_{1} \in \boldsymbol{Z}[\boldsymbol{t}]$. It follows now from (1.0) and (11) that

$$
F^{\prime}\left(X_{1}(\boldsymbol{t}), Y_{1}(\boldsymbol{t})\right)=\frac{A}{N_{\mathfrak{a}}} N \omega_{1}(\boldsymbol{t})=\frac{A}{N_{\mathfrak{l}}} N \gamma_{1} N \omega(\boldsymbol{t})=N_{\mathfrak{c}} \cdot f(\boldsymbol{t})=f(\boldsymbol{t}) .
$$

In the case $c \sim \mathfrak{a}^{2}$ let $\mathfrak{c a}^{-1} \mathfrak{a}^{\prime}=\left(\gamma_{2}\right)$ with $\gamma_{2}$ totally positive and consider the polynomial

We have

$$
\omega_{2}(\boldsymbol{t})=\gamma_{2} \omega(\boldsymbol{t}) \text {. }
$$

$$
\begin{aligned}
O\left(\omega_{2}\right) & =\left(\gamma_{2}\right) C(\omega)=\mathfrak{a n}^{-1} \mathfrak{a}^{\prime} \mathfrak{b} \\
& =\mathfrak{a}^{\prime} \prod_{i=1}^{j} \mathfrak{p}_{i}^{\max \left\{0, a_{i}\right\}+\min \left\{0, b_{i}\right\}} \prod_{i=1}^{j} \mathfrak{p}_{i}^{\prime \max \left\{0, b_{i}\right\}+\min \left\{0, a_{i}\right\}} \prod_{i=1}^{k} q_{i}^{c_{i}},
\end{aligned}
$$

and by $(12) \partial\left(\omega_{2}\right)=0 \bmod a^{\prime}$. 
Since $\left[1, v^{\prime}\right]$ is a basis of $\mathfrak{a}^{\prime}$ we infer that

$$
\omega_{2}(\boldsymbol{t})=X_{2}(\boldsymbol{t})-\vartheta^{\prime} Y_{2}(\boldsymbol{t}),
$$

where $X_{2}, Y_{2} \in Z[t]$. Since $N \gamma_{2}=1$ it follows as before that

$$
F\left(X_{2}(t), Y_{2}(t)\right)=f(t) .
$$

It remains to prove that if there is a form inequivalent to $F$ in the genus of $F$ then $\mathrm{C}$ does not extend to all polynomials $f \in \boldsymbol{Z}[\boldsymbol{t}]$. For this purpose let us observe that there exists then in $\boldsymbol{K}$ a class $C$ of jdeals such that $C^{2}$ is neither the principal class nor the class of $a^{2}$. Choose in $C^{-1}$ a prime ideal $p$ of degree 1 with $N p=p$. There exists a prime ideal $\mathfrak{q}$ such that $\mathfrak{p}^{2} \mathfrak{a q}$ is principal, equal, say $(\alpha)$. Consider the polynomials

$$
\omega(t)=\alpha \frac{t^{p}-t}{p}, \quad f(t)=\frac{A}{N \mathfrak{a}} N \omega(t) .
$$

We have

$$
C(f)=\frac{|A|}{N_{\mathfrak{a}}} \frac{|N \alpha|}{p^{2}}=|A| N_{\mathfrak{q}} \in \boldsymbol{Z}
$$

hence $f(t) \in \boldsymbol{Z}[t]$. Also, since $\frac{t^{p}-t}{p} \in \boldsymbol{Z}$ for all $t \in \boldsymbol{Z}$ we have for all $t \in \boldsymbol{Z}$ : $\omega(t) \in \mathfrak{a} ; \omega\langle t)=x-\vartheta y$ and

$$
f(t)=F(x, y)
$$

for suitable $x, y \in Z$. On the other hand, suppose that

$$
\text { (14) } \quad f(t)=F(X(t), X(t)), \quad X, Y \in \boldsymbol{Z}[t]
$$

and let $x, y$ be the leading coefficients of $X, Y$. Then comparing the leading coefficients on both sides of (14) we get by (13)

$$
\frac{A}{N \mathfrak{a}} \frac{N \alpha}{p^{2}}=F(x, y)=\frac{A}{N \mathfrak{a}} N(x-\vartheta y), \quad N \mathfrak{q}=N \frac{(x-\vartheta y)}{\mathfrak{a}} .
$$

Since $\mathfrak{q}$ is a prime ideal, $x-\vartheta y \in \mathfrak{a}$ it follows that

$$
\frac{(x-\mathfrak{q} y)}{\mathfrak{a}}=q \text { or } \mathfrak{q}^{\prime} \text {. }
$$

Hence $\mathfrak{a} \mathfrak{q} \sim 1$ or $\mathfrak{a} \mathfrak{q}^{-1} \sim 1$. By the choice of $\mathfrak{q}$ this gives $\mathfrak{p}^{2} \sim 1$ or $\mathfrak{p}^{2} \mathfrak{a}^{2} \sim 1$ contrary to the choice of $p$.

Remark. The above proof seems to suggest that if $F^{\prime}$ satisties ( 1 ) and for all $\boldsymbol{t} \in \boldsymbol{Z}^{r}$ the equation (2) is soluble in integers $x, y$ then there exist integer-valued polynomials $X(\boldsymbol{t}), Y(\boldsymbol{t})$ satisfying (3) identically. The following example shows that this is not the case.

$$
\begin{gathered}
\text { Let } F(x, y)=x^{2}+x y+6 y^{2}, \boldsymbol{K}=Q(\sqrt{-23}), \omega=\frac{1+\sqrt{-23}}{2}, \\
f(t)=N\left(\frac{1}{2}\left(\omega^{4}-\omega\right) t^{2}+\omega-8\right)
\end{gathered}
$$

The discriminant of $F$ is -23 hence $F$ is primary. Further, $f(t) \in \boldsymbol{Z}[t]$ since $\left(\frac{1}{2} \omega^{4}-\omega, \omega-8\right)=\frac{(2, \omega)}{\left(2, \omega^{\prime}\right)}$ with $\omega^{\prime}$ conjugate to $\omega$.

Moreover the equation $F(x, y)=f(t)$ is soluble in integers $x, y$ for all $t \in \boldsymbol{Z}$. Indeed if $t \equiv 0 \bmod 2$ we can take

$$
x+y \omega=\left(\frac{1}{2} \omega^{4}-\omega\right) t^{2}+\omega-8
$$

and if $t=1 \bmod 2$ we can take

$$
x+y \omega=\frac{-3-\sqrt{-23}}{-3+\sqrt{-23}}\left[\left(\frac{1}{2} \omega^{4}-\omega\right) t^{2}+\omega-8\right] .
$$

The number on the right-hand side is an integer in $K$ since for $t \equiv 1 \mathrm{mod} 2$

$$
\left(\frac{1}{2} \omega^{4}-\omega\right) t^{2}+\omega-8 \equiv \frac{1}{2} \omega^{4}-8 \bmod 4\left(\omega^{2}-2 \omega\right)
$$

and we have in $\boldsymbol{K}$ the factorizations into prime ideals $(2)=\mathrm{pp}^{\prime},(\omega)=\mathfrak{p} q$, $((-3+\sqrt{-23}) / 2)=p^{3}$.

On the other hand, the polynomial $\left(\frac{1}{2} \omega^{4}-\omega\right) t^{2}+\omega-8$ is irreducible over $\boldsymbol{K}$ since $N \frac{8-\omega}{\frac{1}{2} \omega^{4}-\omega}=\frac{62}{381}$ is not a square in $Q$. Thercfore, if integer-valued polynomials $X(t), Y(t)$ satisfied

$$
F(X(t), X(t))=f(t)
$$

identically, we would have either

or

$$
X(t)+Y(t) \omega=\gamma\left(\frac{1}{2}\left(\omega^{4}-\omega\right) t^{2}+\gamma(\omega-8)\right.
$$

$$
X(t)+X(t) \omega^{\prime}=\gamma\left(\frac{1}{2} \omega^{b}-\omega\right) t^{2}+\gamma(\omega-8)
$$

for some $\gamma \in \boldsymbol{K}$ with $N \gamma=1$. Taking $t=0$ and 1 we would get $\gamma\left(\frac{1}{2} \omega \omega^{4}-\omega, \omega-8\right)$ integral, hence $(\gamma) \frac{\mathfrak{p}}{\mathfrak{p}^{\prime}}$ integral and $(\gamma)=\frac{\mathfrak{p}^{\prime}}{\mathfrak{p}}$. However the ideal on the right-hand side is not principal.

3. IJimma 3. Howy form $I^{\prime}(n, y)$ with at least two distinct aeros can be represented as $F_{1}(a x+b y, a b+d y)$, where $F_{1}$ is primary, $a, b, c, d \in \mathbf{Z}$ and $\left|\begin{array}{ll}a & b \\ c & d\end{array}\right| \neq 0$.

Proof. Suppose that $F(x, y)=G(a x+b y, c x+d y),\left|\begin{array}{ll}a & b \\ c & d\end{array}\right| \neq 0$. Let $I^{*}$ be the product of all distinct primitive irreducible factors of $F$ and similarly $G^{*}$ for $G$. It follows that

$$
F^{*}=C^{-1} G^{*}(a x+b y, a x+d y)
$$


where $O=O\left(G^{*}(a x+b y, e x+d y)\right) \mid C\left(F^{*}\right)$. Hence

$$
\operatorname{dise} F^{*}=O^{2-2\left|F^{*}\right|} \operatorname{disc} G^{*} \cdot\left|\begin{array}{ll}
a & b \\
c & d
\end{array}\right|^{\left[F ^ { * } \left\{\left(\left|F^{*}\right|-1\right)\right.\right.}
$$

and since disc $F^{*} \neq 0,\left|F^{*}\right|>1$ the absolute value of $\left|\begin{array}{ll}a & b \\ c & d\end{array}\right|$ is bounded. Take now a representation of $F(x, y)$ as $G(a x+b y, c x+d y)$, where $\left|\begin{array}{ll}a & b \\ c & d\end{array}\right|$ is maximal. $G$ must be primary, otherwise representing it as $G_{1}\left(a_{1} x+b_{1} y\right.$, $\left.c_{1} w+d_{1} y\right)$ we would obtain a representation of $F$ as $\theta_{1}(\alpha x+\beta y, \gamma x+\delta y)$ with

$$
\operatorname{abs}\left|\begin{array}{ll}
\alpha & \beta \\
\gamma & \delta
\end{array}\right|=\operatorname{abs}\left|\begin{array}{ll}
a & b \\
c & d
\end{array}\right|, \quad \operatorname{abs}\left|\begin{array}{ll}
a_{1} & b_{1} \\
c_{1} & a_{1}
\end{array}\right|>\operatorname{abs}\left|\begin{array}{ll}
a & b \\
c & a
\end{array}\right|
$$

contrary to the choice of $G$, unless $\left|\begin{array}{ll}a_{1} & b_{1} \\ c_{1} & d_{1}\end{array}\right|=0$. In the latter calse however $G$ and hence also $F$ would have only one zero, contrary to the assumption.

Corollary. C implies D.

Proof. Let $F(x, y) \in \boldsymbol{Z}[x, y]$ be any form, $f(\boldsymbol{t}) \in \boldsymbol{Z}[\boldsymbol{t}]$ any polynomial and suppose that for all $\boldsymbol{t} \in \boldsymbol{Z}^{r}$ there exist $x, y \in \boldsymbol{Z}$ satisfying $F(x, y)=f(\boldsymbol{t})$. If $F(x, y)=$ const or $f(\boldsymbol{t})=$ const $\mathrm{D}$ is trivial. If $F(x, y)$ has only one zero, we take without loss of generality $F^{\prime}(x, y)=a(b x+c y)^{n}$, where $b \neq 0$. Applying Theorem 3 of [13] to the eduation $a u^{n}=f(t)$ we infer the existence of a polynomial $U(\boldsymbol{t}) \in \boldsymbol{Q}[\boldsymbol{t}]$ such that a $U(\boldsymbol{t})^{n}=f(\boldsymbol{t})$. It suffices to take $X(\boldsymbol{t})=b^{-1} U(\boldsymbol{t}), Y(\boldsymbol{t})=0$.

If $F^{\prime}(x, y)$ has at least two distinct zeros then by Lemma $3 \cdot F^{\prime}(x, y)$ $=F_{x}(a x+b y, a x+d y)$, where $F_{1}$ is primary and $\left|\begin{array}{ll}a & b \\ c & d\end{array}\right| \neq 0$. On the other hand there exists a vector $\boldsymbol{t}_{0} \in \boldsymbol{Z}^{r}$. such that $f\left(\boldsymbol{t}_{0}\right)=e \neq 0$. Oonsider now the equation

$$
F_{I}(x, y)=f\left(e t+t_{0}\right)
$$

The polynomial on the right-hand side has both the content and the fixed divisor equal to $|e|$, hence by $\mathrm{C}$ there exist polynornials $X_{I}, Y_{I} \in \mathbb{Z}[\boldsymbol{t}]$ such that $F_{1}\left(X_{1}(t), Y_{1}(t)\right)=f\left(e t+t_{0}\right)$. Dotermining $X(t), Y(t)$ from the equations

$$
\begin{aligned}
& a X(\boldsymbol{t})+b Y(\boldsymbol{t})=X_{1}\left(\frac{\boldsymbol{t}-\boldsymbol{t}_{0}}{e}\right) \\
& a X(\boldsymbol{t})+a X(\boldsymbol{t})=Y_{1}\left(\frac{\boldsymbol{t}-\boldsymbol{t}_{0}}{e}\right)
\end{aligned}
$$

we get

$$
X(\boldsymbol{t}), X(\boldsymbol{t}) \Subset Q[\boldsymbol{t}], \quad W(X(t), X(t))=f(\boldsymbol{t}),
$$

Limana 4. H implies the following.

Let $f_{v} \in \boldsymbol{Z}[\boldsymbol{t}](1 \leqslant v \leqslant n)$ be distinot irreducible polynomials such that their leading forms $h_{v}(\boldsymbol{t})$ all assume a positive value for $a \boldsymbol{t} \in \boldsymbol{N}^{r}$ and that $\prod_{v=1}^{n} f_{\nu}(\boldsymbol{t})$ has the fixed divison 1. Then for any $B$ there exists a $\boldsymbol{t} \in \mathbf{N}^{r}$ such that $f_{\eta}(\boldsymbol{t})$ are alstinct primes $>B$.

Proof. The condition. that $f_{p}$ are irreducible and distinct implies that they are prime to each other. Indeed, otherwise two of them would differ by a constant factor $\theta \neq 1$. The numerator and the denominator of $\theta$ would divide $\prod_{p=1}^{n} f_{v}(\boldsymbol{t})$ for all $t$ bence $o=-1$. But this contradicts the condition on $h_{y}{ }^{p}$.

Let us choose an $a \in N^{r}$ such that

and let

$$
h_{y}(\boldsymbol{a})>0 \quad(1 \leqslant \nu \leqslant n)
$$

$$
a=\left(\left|h_{1}\right|+\left|h_{2}\right|+\ldots+\left|h_{p}\right|\right) ! \prod_{\nu=1}^{n} h_{p}(\boldsymbol{a})
$$

Since

$$
f(\boldsymbol{t})=\prod_{\nu=\mathbb{1}}^{n} f_{v}(\boldsymbol{t})
$$

has the fixed divisor 1 we infer from the Chinese Remainder Theorem the existence of a $\tau \in \mathbb{Z}^{r}$ such that

$$
(f(\tau), a)=1 \text {. }
$$

Consider the polynomials $f_{v}(a x+a t+\tau)(1 \leqslant v \leqslant n)$.

They are irreducible as polynomials in $x, t$ and primse to each other. Consequently the resultant $R_{\mu, v}(\boldsymbol{t})$ of $f_{\mu}(a x+a t+\tau)$ and $f_{\nu}(a x+a t+\tau)$ is non-zero for all $\mu<\nu \leqslant n$. By Hilbert's irreducibility theorem there exists a $t_{0} \in Z^{r}$ such that $f_{\nu}\left(a x+a t_{0}+\tau\right)(1 \leqslant \nu \leqslant n)$ are all irreducible as polynomials in an and

$$
\prod_{\mu<\mu}^{n} R_{\mu, \mu}\left(\boldsymbol{t}_{0}\right) \neq 0
$$

The loading coefficintis of $f_{11}\left(a x+a t_{0}+\tau\right)$ aro positive by (15). Moroover

$$
p(x)=\prod_{y=1}^{16} f_{p}\left(a n+a t_{0}+x\right)
$$

has the lixed divisor 1. Indeed, the $|p|$ th difference

$$
d^{|x|} p(0)=a
$$


on the other hand

$$
p(0)=f\left(a t_{0}+\tau\right)=f(\tau) \bmod a
$$

and we get $\left(p(0), \Delta^{|p|}(0)\right)=1$ by $(16)$.

By $\boldsymbol{E}$ there exist infinitely many $x \in \boldsymbol{N}$ such that $f_{\nu}\left(\boldsymbol{a} x+a \boldsymbol{t}_{0}+\tau\right)$ are primes. For sufficiently large $x$ we have $a x+a t_{0}+\tau \in \boldsymbol{N}^{r}$ and

$$
f_{\nu}\left(\boldsymbol{a} x+a t_{0}+\tau\right)>|B|+\sum_{\mu<\nu}^{n}\left|R_{\mu, \nu}\left(\boldsymbol{t}_{0}\right)\right|
$$

Thus the primes in question are $>B$. They are distinet since the common value of $f_{\mu}\left(a x+a t_{0}+\tau\right)$ and $f_{\nu}\left(a x+a t_{0}+\tau\right)$ would have to divide $R_{\mu, \nu}\left(t_{0}\right)$ which is impossible by (17) and (18).

LEMma 5. Let $\boldsymbol{K}$ be the rational field or a quadratic field, $\Lambda$ be the discriminant of $\boldsymbol{K}$ and let $\varphi_{v} \in \boldsymbol{K}[\boldsymbol{t}](1 \leqslant \nu \leqslant n)$ be polynomials irreduoible over $\boldsymbol{K}$ and prime to each other. If

$$
\text { (19) the fixed divisor of } \prod_{\nu=1}^{n} N \varphi_{\nu}(\boldsymbol{t}) \text { equals } \prod_{\nu=1}^{n} N O\left(\varphi_{\nu}\right)
$$

then for every $M \in \boldsymbol{N}$, there exists a $\mu \in \boldsymbol{N}$ prime to $M$ with no prime ideal factor of degree 1 in $\boldsymbol{K}$ and $\tau \in \boldsymbol{Z}^{r}$ with the following property. Let

$$
\psi_{\nu}(\boldsymbol{t})=\varphi_{\nu}(\mu \boldsymbol{t}+\tau) \quad(1 \leqslant \nu \leqslant n) .
$$

For any $A \in \boldsymbol{N}, \boldsymbol{t}_{1} \in \boldsymbol{Z}^{r}$ and $m \in \boldsymbol{N}$ prime to $\Delta \prod_{\eta_{n=1}^{n}}^{n} \frac{N \psi_{\nu}\left(\boldsymbol{t}_{1}\right)}{N O\left(\psi_{\nu}\right)} \mathrm{H}$ implies the existence of $a \boldsymbol{t}_{2} \in \boldsymbol{N}^{r}$ such that $\boldsymbol{t}_{2} \equiv \boldsymbol{t}_{1} \bmod m$, all the ideals $\frac{\left(\psi_{n}\left(\boldsymbol{t}_{2}\right)\right)}{C\left(\psi_{v}\right)}$ are prime in $\boldsymbol{K}$, distinct and do not divide $A$.

Moreover, either $\mu=1, \tau=0$ have the above property (this happens for $\boldsymbol{K}=Q$ ) or there is a sequence of pairs $\left\langle\mu_{i}, \tau_{i}\right\rangle$ with the above property sueh that $\left(\mu_{i}, \mu_{h}\right)=1$ for $i \neq h$, and the number of distinat $\mu_{i} \leqslant x$ is greater than $c x^{1 / n} / \log x$ for a certain $c>0$ and all $x>x_{0}$.

Proof. We begin with a remark concerning the fixed divisor that we shall use twice. If $P \in Z[t]$ has the fixed divisor $d$ then any fixed prime divisor $p$ of $P(m t+a)$ divides $d m$. Indeed if $p \nmid d$ then there exists a $u \in Z^{r}$ such that $P(\boldsymbol{u}) \neq 0 \bmod p$ and if $p \nmid m$ there cxists a $v \in Z^{r}$ such that $m \boldsymbol{v}+\boldsymbol{a} \equiv \boldsymbol{u} \bmod p$, hence $P(m \boldsymbol{v}+\boldsymbol{a}) \neq 0 \bmod p$.

Now we proceed to the proof of the lemma. Let

$$
\begin{gathered}
\phi_{\nu}(\boldsymbol{t})=a_{\nu} f_{\nu}(\boldsymbol{t}) \quad(\nu \leqslant k), \\
N \varphi_{\nu}(\boldsymbol{t})=a_{v} f_{\nu}(\boldsymbol{t}) \quad(k<\nu \leqslant n),
\end{gathered}
$$

where $f_{v} \in \boldsymbol{Z}[\boldsymbol{t}]$ are irreducible over $\boldsymbol{Q}$ and

$$
\begin{gathered}
\left(a_{\nu}\right)=C\left(p_{\nu}\right) \quad(\nu \leqslant k), \\
\left|a_{\nu}\right|=N C\left(p_{\nu}\right) \quad(k<\nu \leqslant n) .
\end{gathered}
$$

(If $\boldsymbol{K}=\boldsymbol{Q}$ we take $\bar{k}=\mathbf{0}$.) Let $h_{v}$ be the leading form of $f_{v}$. We can choose the signs of $a_{v}$ so that for a suitable $\boldsymbol{t} \in \boldsymbol{N}^{r}: h_{v}(\boldsymbol{t})>0$ for all $\nu \leqslant n$. We have

$$
\prod_{\nu=1}^{n} \frac{N \varphi_{\nu}(\boldsymbol{t})}{N C\left(\varphi_{\nu}\right)}= \pm \prod_{\nu=1}^{k} f_{\nu}^{2}(\boldsymbol{t}) \prod_{\nu=1}^{n} f_{\nu}(\boldsymbol{t})
$$

and (19) implies on an application of the Chinese Remainder Theorem that for a suitable $\tau_{0} \in \boldsymbol{Z}^{r}$

$$
\left(\Delta, \prod_{p=1}^{n} f_{p}\left(\tau_{0}\right)\right)=1
$$

Let $f_{\nu}\left(\tau_{0}\right)=\varrho_{\nu} \bmod A, \varrho_{\nu}>0(\nu \leqslant \hbar)$. Without loss of generality we may assume that

$$
\text { (22) } \quad\left(\frac{\Delta}{\varrho_{\nu}}\right)=1(1 \leqslant \nu \leqslant j), \quad\left(\frac{\Delta}{\varrho_{\nu}}\right)=-1(j \leqslant \nu \leqslant k) .
$$

Since $\varphi_{y}$ are prime to each other

(23) $\quad\left(f_{\lambda}, f_{v}\right)=1 \quad$ unless $\lambda=\nu$ or $\lambda>k, v>k$ and $\varphi_{\lambda} / \varphi_{p}^{\prime} \in \boldsymbol{K}$ where $p_{v}^{\prime}$ is conjugate to $\varphi_{v}$ over $\boldsymbol{Q}(\boldsymbol{t})$.

In particular, $f_{1}, \ldots, f_{j}$ and $\prod_{\nu=j+1}^{n} f_{\nu}$ are prime to each other. Let $\boldsymbol{t}=\left[\begin{array}{ll}t, \boldsymbol{t}^{\prime}\end{array}\right], a_{0}\left(\boldsymbol{t}^{\prime}\right)$ be the leading coefficient of $\prod^{n} f_{\nu}(\boldsymbol{t}), D\left(\boldsymbol{t}^{\prime}\right)$ the discriminant of $\prod_{\nu=1}^{j} f_{v}(\boldsymbol{t})$ and $R\left(\boldsymbol{t}^{\prime}\right)$ the resultant of $\prod_{\nu=1}^{j} f_{p}(\boldsymbol{t}), \prod_{\nu=j+1}^{n} f_{\nu}(\boldsymbol{t})$ with respect to $t$. It follows that

$$
a_{0} D R \neq 0 \text {. }
$$

Since $f_{n}(\boldsymbol{t})$ are irreducible over $\boldsymbol{K}$ for $\nu \leqslant j$ we infer by Hilbert's irreducibility theorem that there exists a $\boldsymbol{\tau}^{\prime} \in Z^{r-1}$ such that $f_{v}\left(t, \boldsymbol{\tau}^{\prime}\right)$ are irreducible over $\boldsymbol{K}$ for $y \leqslant j$ and

$$
\text { (25) } \quad a_{0}\left(\tau^{\prime}\right) D\left(\tau^{\prime}\right) R\left(\tau^{\prime}\right) \neq 0 \text {. }
$$

Let $f_{\nu}\left(\vartheta_{\nu}, \tau^{\prime}\right)=0$ and $\boldsymbol{K}_{p}=\boldsymbol{Q}\left(\vartheta_{\nu}\right)(\nu \leqslant j)$. We have $\boldsymbol{K} \leftarrow \boldsymbol{K}_{\nu}$ and by Bauer's theorem thore exist for each $v \leqslant j$ infinitely many primes with a prime ideal factor of degree 1 in $\boldsymbol{K}_{y}$, but not in $\boldsymbol{K}$. Choose for each $\nu \leqslant j$ a different prime $p_{p}$ with the above property and such that

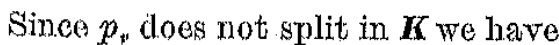

$$
\left(\frac{\Delta}{p_{p}}\right)=-1 \quad(\nu \leqslant j) .
$$

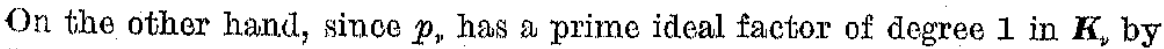
Dedokind's theorem there exists an integer $u$ such that

$$
f_{\nu}\left(u, \tau^{\prime}\right)=0 \bmod p_{p} .
$$


By (25) and (26) the discriminant of $\prod_{i=1}^{j} f_{i}\left(t, \tau^{\prime}\right)$ equals,$D\left(\tau^{\prime}\right) \neq 0\left(\bmod p_{\eta}\right)$. Since $a_{0}\left(\tau^{\prime}\right) \not \equiv 0 \bmod p_{v}$ and $\prod_{i=1}^{j} f_{i}\left(\iota, \tau^{i=1} \equiv 00 \bmod p_{\nu}\right.$ we infer from Lemma 1 that either

$$
\prod_{i=1}^{j} f_{i}\left(u, \tau^{\prime}\right) \not 0 \bmod p_{n}^{2}
$$

or

$$
\prod_{i=1}^{j} f_{i}\left(u+p_{v}, \tau^{\prime}\right) \neq 0 \bmod p_{v}^{2} .
$$

Therefore, there exists an integer $\tau_{p}$ such that

$$
\begin{aligned}
f_{v}\left(\tau_{v}, \tau^{\prime}\right) & \equiv 0 \bmod p_{v}, \\
\prod_{i=1}^{j} f_{i}\left(\tau_{v}, \tau^{\prime}\right) & \equiv 0 \bmod p_{v}^{2} .
\end{aligned}
$$

Moreover, since by (25) and (26) the resultant of $\prod_{i=1}^{j} f_{i}\left(t, \tau^{\prime}\right)$ and $\prod_{i=j_{+1}}^{n} f_{i}\left(t, \tau^{\prime}\right)$ equal to $R\left(\tau^{\prime}\right) \not \equiv 0 \bmod p_{v}$ we have

$$
\prod_{i m j+1}^{n} f_{i}\left(\tau_{v}, \tau^{\prime}\right) \neq 0 \bmod p_{v} .
$$

Let us choose $\tau \equiv \tau_{\nu} \bmod p_{v}^{2}(1 \leqslant \nu \leqslant j)$ and set

$$
\mu=\prod_{v=1}^{j} p_{v}, \quad \tau=\left[\tau, \tau^{\prime}\right]
$$

By (28)-(30) we have

$$
\begin{gathered}
f_{p}(\tau)=0 \bmod p_{p}, \\
\prod_{i=1}^{n} f_{i}(\tau) \neq 0 \bmod p_{\nu}^{2} .
\end{gathered}
$$

We shall show that

$$
\prod_{i=1}^{n t} f_{i}(\mu \boldsymbol{t}+\tau)=P^{\prime}(\mu \boldsymbol{t}+\tau)
$$

has the fixed divisor $d$ equal to $p_{1} p_{2} \ldots p_{j}$. Indeed by $(19)$ and $(20)$ the fixed divisor of $P(\boldsymbol{t})$ equals 1 , hence a consists of prime factors of $\mu$. Kowever by (33)

$$
d \not 0 \bmod p_{v}^{2} \quad(\nu \leqslant j) .
$$

On the other hand by (31) and (32)

$$
f_{p}(\mu t+\tau) \equiv f_{y}(\tau)=0 \bmod p_{v} .
$$

Thus $d=p_{1} p_{2} \ldots p_{j}$, the polynomials

$$
\begin{aligned}
& g_{\nu}(\boldsymbol{t})=p_{\nu}^{-1} f_{\nu}(\mu \boldsymbol{t}+\tau) \quad(\nu \leqslant j), \\
& g_{\nu}(\boldsymbol{t})=f_{\nu}(\mu \boldsymbol{t}+\tau) \quad(j<\nu \leqslant n)
\end{aligned}
$$

have integral coefficients, $\prod_{v=1}^{n} g_{v}(t)$ has the fixed divisor 1 and a fortiori the content 1. Moreover by $(23)$

(35) $\quad g_{\lambda} \neq g_{v}$ unless $\lambda=v$ or $\lambda>k, \nu>k$ and $\varphi_{\lambda} / p_{v}^{\prime} \in \boldsymbol{K}$.

It follows that

where besides

$$
\begin{array}{cc}
\psi_{\nu}(\boldsymbol{t})=a_{v} p_{\nu} g_{\nu}(\boldsymbol{t}) & (\nu \leqslant j), \\
\psi_{\nu}(\boldsymbol{t})=a_{\nu} g_{\nu}(\boldsymbol{t}) & (j<\nu \leqslant k), \\
N \psi_{\nu}(\boldsymbol{t})=a_{\nu} g_{\nu}(\boldsymbol{t}) & \left(h_{i}<\nu \leqslant n\right),
\end{array}
$$

(38) $\quad O\left(\psi_{v}\right)=\left(a_{v} p_{v}\right)(v \leqslant j), \quad C\left(\psi_{v}\right)=\left(a_{v}\right)(j<\nu \leqslant l k)$,

(39) $\quad N C\left(\psi_{v}\right)=\left|a_{v}\right| \quad(k<\nu \leqslant n)$.

It follows that

$$
\prod_{v=1}^{n} \frac{N \psi_{v}(\boldsymbol{t})}{N O\left(\psi_{v}\right)}= \pm \prod_{p=1}^{k} g_{p}^{2}(\boldsymbol{t}) \prod_{p=k+1}^{n} g_{p}(\boldsymbol{t}) .
$$

If now for a $\boldsymbol{t}_{\mathbf{1}} \in \boldsymbol{Z}^{r}$ we have

$$
\left(m, \Delta \prod_{v=1}^{n} \frac{N p_{v}\left(\boldsymbol{t}_{1}\right)}{N C\left(\psi_{v}\right)}\right)=1
$$

there existis a $\boldsymbol{t}_{0} \in \boldsymbol{Z}^{r}$ satisfying

$$
\boldsymbol{t}_{0}=\boldsymbol{t}_{1} \bmod m, \quad \boldsymbol{\mu} \boldsymbol{t}_{0}+\boldsymbol{\tau} \equiv \boldsymbol{\tau}_{0} \bmod \Delta .
$$

Since

$$
\left(m, \prod_{n=1}^{n}\left[g_{v}\left(t_{0}\right)\right)=\left(m, \prod_{i=1}^{n} g_{p}\left(t_{1}\right)\right)=1\right.
$$

and by (34) and (21)

$$
\left(A, \prod_{v=1}^{n} g_{v}\left(t_{0}\right)\right)=\left(\Delta, \prod_{p=1}^{n} g_{p}(0)\right)=\left(\Delta, \prod_{\nu=1}^{n} f_{\nu}\left(\tau_{0}\right)\right)=1
$$

it follows that

$$
\prod_{p=1}^{n} g_{v}\left(\Delta m \boldsymbol{t}+\boldsymbol{t}_{0}\right)
$$


has the fixed divisor 1. The polynomials $g_{\nu}\left(\Delta m \boldsymbol{t}+\boldsymbol{t}_{0}\right)$ are irreducible and their leading forms all take a positive value for a suitable $\boldsymbol{t} \in \boldsymbol{N}^{r}$ in virtue of the corresponding property of $f_{v}(\boldsymbol{t})$. By Lemma $4 \mathrm{H}$ implies the existence of an $\boldsymbol{x} \in \boldsymbol{N}^{r}$ such that $g_{v}\left(\Delta m \boldsymbol{x}+\boldsymbol{t}_{0}\right)$ are primes greater than $|A|$ and

$$
g_{\lambda}\left(\Delta m \boldsymbol{x}+\boldsymbol{t}_{0}\right) \neq g_{v}\left(\Delta m \boldsymbol{x}+\boldsymbol{t}_{0}\right) \text { unless } g_{\lambda}=g_{v} .
$$

Taking $\boldsymbol{t}_{2}=\Delta m \boldsymbol{x}+\boldsymbol{t}_{0}$ we get from (40)

$$
\boldsymbol{t}_{2} \equiv \boldsymbol{t}_{1} \bmod m, \quad \mu \boldsymbol{t}_{2}+\tau \equiv \tau_{0} \bmod \Delta
$$

Thus by (34)

$$
\begin{gathered}
p_{v} g_{\nu}\left(\boldsymbol{t}_{2}\right)=f_{v}\left(\mu \boldsymbol{t}_{2}+\tau\right) \equiv f_{\nu}\left(\tau_{0}\right) \equiv \varrho_{v} \bmod \Delta \quad\langle\nu \leqslant j), \\
g_{v}\left(\boldsymbol{t}_{2}\right)=f_{v}\left(\mu \boldsymbol{t}_{2}+\tau\right) \equiv f_{v}\left(\boldsymbol{\tau}_{0}\right) \equiv \varrho_{\nu} \bmod \Delta \quad(j<\nu \leqslant 7)
\end{gathered}
$$

and we infer from (22) and (27) that

$$
\left(\frac{\Delta}{g_{v}\left(\boldsymbol{t}_{2}\right)}\right)=-1 \quad(\nu \leqslant k)
$$

Hence for $v \leqslant k g_{v}\left(\boldsymbol{t}_{2}\right)$ are prime in $\boldsymbol{K}$ not dividing $A$ and in virtwe of (36) and (38) the same applies to the ideals $\mathfrak{a}_{p}=\frac{\left(\psi_{v}\left(\boldsymbol{t}_{2}\right)\right)}{\partial\left(\psi_{p}\right)}$. The remaining ideals $\mathfrak{a}_{y}(\nu<k \leqslant n)$ are prime and do not divide $A$ in virtue of (37) and (39). Assuming

$$
\lambda \neq \nu, \quad \mathfrak{a}_{\lambda}=\mathfrak{a}_{\nu}
$$

we get by (35) and (41) for a suitable $\gamma \in \boldsymbol{K}$

$$
\begin{gathered}
\lambda>k, \quad \nu>k, \quad \varphi_{\lambda}=\gamma \varphi_{\nu}^{\prime}, \quad \psi_{\lambda}=\gamma \psi_{\nu}^{\prime}, \quad C\left(\psi_{2}\right)=(\gamma) O\left(\psi_{\nu}^{\prime}\right), \\
\frac{\left(\psi_{\nu}\left(\boldsymbol{t}_{2}\right)\right)}{O\left(\psi_{\nu}\right)}=\frac{\left(\psi_{\nu}^{\prime}\left(\boldsymbol{t}_{2}\right)\right)}{C\left(\psi_{\nu}^{\prime}\right)},
\end{gathered}
$$

thus the ideal $\mathfrak{a}_{,}$is ambiguous.

By Dedekind's theorem $\mathfrak{a}_{\mathfrak{y}} \mid \Delta$, hence by $(37)$ and (39)

However by (34) and (42)

$$
g_{v}\left(\boldsymbol{t}_{2}\right) \mid A \text {. }
$$

$$
g_{v}\left(\boldsymbol{t}_{2}\right)=f_{p}\left(\mu \boldsymbol{t}_{2}+\tau\right) \equiv f_{p}\left(\tau_{0}\right) \bmod \Delta
$$

and we get a contradiction with (21). The contradiction shows that the ideals $\mathfrak{a}_{v}$ are distinct and the proof of the first part of the lemma is complete.

To prove the second part we note that if $j=0$ (31) gives $\mu=1$. The value of $\tau$ is then irrelevant and can be taken 0 . Therefore assume that $j>0$ and that we have already defined $\left\langle\mu_{1}, \tau_{i}\right\rangle, \ldots,\left\langle\mu_{i-1}, \tau_{i-1}\right\rangle$ $(i \geqslant 1)$, each $\mu_{i}$ with $j$ prime factors. Then we replace in the above proof $M$ by $M \mu_{1} \ldots \mu_{i-1}$ and define $\mu_{i}, \tau_{i}$ by (31). It is clear that the sequence thus obtained satisfies $\left(\mu_{i}, \mu_{h}\right)=1$ for $i \neq h$. Denote by $P\left(\boldsymbol{K}_{p}\right)$ the set of primes with a prime ideal factor of degree 1 in $\boldsymbol{K}_{\nu}$. By Bauer's theorem $P\left(\boldsymbol{K}_{v}\right) \backslash P(\boldsymbol{K})$ has a positive density, say, $\delta_{v}$. Computing $\mu_{i}$ from (31) we take $p_{v}$ to be the least element of $P\left(\boldsymbol{K}_{v}\right) \backslash P(\boldsymbol{K})$ different from $\omega+j(i-1)+y-1$ given primes, where $\omega$ is the number of prime factors of $M a_{0}\left(\tau^{\prime}\right) D\left(\tau^{\prime}\right) R\left(\tau^{\prime}\right)$. Hence for $i>i_{0}$ we have $p_{v} \leqslant 2 \delta_{y}^{-1} j i \log j i$ and

$$
\mu_{i}=\prod_{p=1}^{j} p_{p} \leqslant\left(e^{-1} j i \log j i\right)^{j}, \quad e=\frac{1}{2} \prod_{v=1}^{j} \delta_{v}^{1 / j} .
$$

Since the number of solutions of the inequality

$$
\left(c^{-1} j i \log j i\right)^{j} \leqslant x
$$

in positive integers $i$ is for $x$ large enough at least $\frac{a x^{1 / 3}}{\log x-1}$, the number of distinct $\mu_{i} \leqslant x$ is at least

$$
\frac{c x^{1 / j}}{\log x-1}-i_{0}>\frac{c x^{1 / n}}{\log x} \quad\left(x>x_{0}\right)
$$

which completes the proof.

Remark. The lomma extends to all cyclic fields.

LIEMMA 6. Let $\boldsymbol{K}$ be any field, $f \in \boldsymbol{K}[\boldsymbol{t}]$ a non-zero polynomial. If a form $F^{7} \in \boldsymbol{K}[x, y]$ has at least three distinot zeros in the algebrais olosure of $\boldsymbol{K}$ then there exist no more than $\left.\left|F^{3}\right|^{3}\right|^{|f|}$ pairs $\langle X(\boldsymbol{t}\rangle, Y(\boldsymbol{t})\rangle$ such that $X, Y \in \boldsymbol{K}(\boldsymbol{t})$, $X, Y$ linearly independent over $\boldsymbol{K}$ and

$$
Z^{\prime}(X(\boldsymbol{t}), X(\boldsymbol{t}))=f(\boldsymbol{t}) .
$$

Proof. Without loss of generality we may assume that $\boldsymbol{K}$ is algebraically closed. By a linear transformation we can transform $F$ to the form

$$
F^{\prime}(x, y)=x^{l} y^{l} G(x, y), \quad k \geqslant 1, l \geqslant 1, \quad(G(x, y), x y)=1 .
$$

Let us assign two solutions $\left\langle X_{1}, Y_{1}\right\rangle$ and $\left\langle X_{2}, Y_{2}\right\rangle$ of (43) to the same class if $X_{2}=\xi X_{1}, X_{2}=\eta X_{1}$ for some $\xi, \eta \in \boldsymbol{K} \backslash\{0\}$. The number of elasses does not ( $x$ ured tho number of pairs of monic polynomials $x, y \in \mathbf{K}[\boldsymbol{t}]$ such that

$$
x y \mid f(t)
$$

which is clearly bounded by $3^{|f|}$. The number of polynomials in one class can be estimated as follows.

$$
\text { If }
$$

then

$$
\Pi\left(\xi X_{1}, \eta Y_{1}\right)=W\left(X_{1}, Y_{1}\right)
$$

$$
F\left(\xi \frac{X_{1}}{Y_{1}}, \eta\right)=F\left(\frac{X_{1}}{Y_{1}}, 1\right)
$$


and since $X_{1} / Y_{1}$ takes in $\boldsymbol{K}$ infinitely many values we have identically Hence

$$
F(\xi u, \eta)=F(u, 1) \text {. }
$$

$$
\xi^{k} \eta^{l} G(\xi u, \eta)=G(\imath, 1)
$$

and the comparison of the leading coefficients and of the constant terms on both sides gives

It follows that

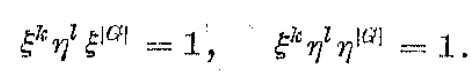

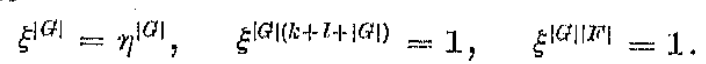

Thus there are $|G||F|$ possibilities for $\xi$ and for each $\xi$ at most $|G|$ possibilities for $\eta$, which gives at most $\left|F^{\prime}\right|\left|G^{q}\right|^{2} \leqslant\left|F^{3}\right|^{3}$ possibilities for $\langle\xi, \eta\rangle$. The lemma follows.

Lisnma 7 . If $F(x, y) \in \boldsymbol{Z}[x, y]$ is a non-singular oubio form then for every integer $a \neq 0$ the number of solutions of the equation $F(x, y)=a z^{3}$ in integers $x, y, z$ such that $\langle x, y, z)=1$ and $1 \leqslant z \leqslant Z$ is $O\left((\log Z)^{b}\right)$, where $b$ is a constant depending on $F^{\prime}$ and $a$.

Proof. It is enough to estimate the number of solutions with $|x| \leqslant|y|$. Assume that

$$
F(x, y)=a z^{3}, \quad 1 \leqslant z \leqslant Z \text { and }|x| \leqslant|y| .
$$

If $W^{\prime}(1,0)=0$ we have $|F(x, y)| \geqslant|y|$ hence $h=\max (|x|,|y|,|z|) \ll Z^{3}$, where the constant in the symbol $\ll$ depends on $a$, later also on $f$. If $F(1,0) \neq 0$ let

$$
F^{\prime}(x, y)=a_{0} \prod_{l=1}^{3}\left(x-\xi_{l} y\right),
$$

where $\xi_{1}$ is the real zero of $W^{\prime}$ nearest to $x / y$. Since $F(x, y) \neq 0$ we have by Thue's theorem

$$
\left|x-\xi_{1} y\right| \gg|y|^{-3 / 2} \text {. }
$$

On the other hand $\left|x-\xi_{2} y\right|\left|x-\xi_{3} y\right| \gg y^{2}$. Henee by (4t4) and (45)

$$
|a| z^{3}=|F(x, y)| \gg y^{1 / 2} \quad \text { and } \quad h \ll Z^{6} .
$$

Since $F(x, y)=a z^{3}$ represents in projective coordinates a curve of genus 1 , in virtue of a theorem of Néron (see [8], p.82) the number of solutions of (44) is $O\left(\left(\log Z^{6}\right)^{g / 2+1}\right)$ where $g$ is the rank of the curve.

Remark. The lemma extends to all forms $F$ with at least three distinct zeros. If the genus of the curve $f^{\prime}(x, y)=a z^{\left|F^{\prime}\right|}$ is greater than 1 one needs a theorem of Mumford [10].

Limma 8. Let $\boldsymbol{K}$ be any field, $U$ a finite subset of $\boldsymbol{K}$ and $P \in \boldsymbol{K}[\boldsymbol{t}]$, $P \neq 0$. The equation $P(\boldsymbol{t})=0$ has no more than $|P||U|^{r-1}$ solutions $\boldsymbol{t} \in U^{r}$, where $|U|$ is the number of elements of $U$.
Proof (by induction on $r$ ). For $r=1$ the assertion is obvious. Assume that it holds for polynomials in $r-1$ variables and let

$$
P(\boldsymbol{t})=\sum_{i=0}^{p} P_{\boldsymbol{i}}\left(\boldsymbol{t}^{\prime}\right) t_{1}^{p-1}
$$

The solutions of $P(\boldsymbol{t})=0$ are of two kinds: satisfying $P_{0}\left(\boldsymbol{t}^{\prime}\right)=0$ and $P_{0}\left(\boldsymbol{t}^{\prime}\right) \neq 0$. Since $t_{1}$ can take at most $|U|$ values, by the inductive assumption the number of solutions of the first kind does not exceed $\left|P_{0}\right||\sigma|^{-1-1}$. Similarly since $\boldsymbol{t}^{\prime}$ can talke at most $|U|^{r-1}$ values the number of solutions of tillo second kind does not exceed $p|U|^{r-1}$. However $\left|P_{0}\right|+p \leqslant|P|$ and the proof is complete.

Remark. A different proof can be obtained by an adaptation of the proof given by Schmidt for the special case $\boldsymbol{K}=U$ (see [17], p. 147, Lemma 3A).

IInuma 9. If $f(\boldsymbol{t}), g(\boldsymbol{t}) \in \boldsymbol{Q}[\boldsymbol{t}], g(\boldsymbol{t}) \mid f(\boldsymbol{t})^{n}$ and the fixed divisor of $f(\boldsymbol{t})$ equals $O(f)$ then the fixed divisor of $g(\boldsymbol{t})$ equats $O(g)$.

Proof. Teet the fixed divisor of $g$ be $C(g) d, d \in \boldsymbol{N}$ and let $f(\boldsymbol{t})^{n}=g(\boldsymbol{t}) h(\boldsymbol{t})$. Clearly for all $\boldsymbol{t} \in \boldsymbol{Z}^{r} f(\boldsymbol{t})^{n}$ is divisible by $O(g) d O(h)=d O\left(f^{n}\right)=d O(f)^{n}$ On the other hand the fixed divisor of $f(t)^{n}$ is $O(f)^{n}$. Hence $d=1$.

Proof of Theorew 2. Consider first the case, where $F$ is ar quadratic form. Then by Lemma 2

$$
F^{\prime}(x, y)=A\left(a x^{2}+b x y+o y^{2}\right), \quad \text { where } \quad A, a, b, c \in \boldsymbol{Z}
$$

and either $\Delta=b^{2}-4 a c=1$ or $\Delta$ is a fundamental discriminant. Since the tixed divison of $f(t)$ equals $C(f)$ we have $A \mid O(f)$ and we can assume without loss of generality that $A=1$. Let $K=Q(\sqrt{4})$

$$
f(\boldsymbol{t})=l \prod_{p=1}^{n} \varphi_{v}(\boldsymbol{t})^{e_{y}}
$$

be a factorization of $f(\boldsymbol{t})$ over $\boldsymbol{K}$ into irreducible factors such that $\varphi_{\text {p }}$ are distinct and have the coefficient of the first term in the antilexicographio order equal to 1 . Since the fixed divisor of $f$ equals $C(f)$ the condition (19) is satisfied in virtuce of Lemma 9 . Let $\mu, \tau$ be parameters whose existence for $\left\{p_{p}\right\}$ and $M=a$ is asserted in Lemma 5 and let

It follows that

$$
\psi_{\nu}=p_{\nu}(\mu t+\tau) \quad(1 \leqslant \nu \leqslant n) .
$$

and

$$
f(\mu t+\tau)=l \prod_{p=1}^{n} \psi_{\nu}(\boldsymbol{t})^{e_{*}}
$$

$$
B=\mid \eta_{i} \prod_{\nu=1}^{n} C\left(\psi_{\nu}\right)^{e_{y}}=O(f(\mu \boldsymbol{t}+\tau)) \in \boldsymbol{N},
$$


where an ideal in $Q$ is identified with its positive generator. If $A=1$ is equivalent to $x y$ and Theorem 1 applies. Assume that $A \neq 1$, thus is a quadratic field. Taking $m=1$ in Lemma 5 we infer that $\mathrm{H}$ impli the existence of a $\boldsymbol{t}_{2} \in \boldsymbol{Z}^{r}$ such that $\frac{\left(\psi_{p}\left(\boldsymbol{t}_{2}\right)\right)}{\sigma\left(\psi_{p}\right)}$ are distinct prime ides of $\boldsymbol{K}$ not dividing $B$. By the assumption there exist $x_{0}, y_{0} \in \boldsymbol{Z}$ such th

$$
a x_{0}^{2}+b x_{0} y_{0}+o y_{0}^{2}=f\left(\mu t_{2}+\tau\right)
$$

Hence, after a transformation

$$
N \frac{\left(a x_{0}+\frac{b+\sqrt{\Delta}}{2} y_{0}\right)}{\mathfrak{a}}=\left|f\left(\mu \boldsymbol{t}_{2}+\tau\right)\right|, \quad \text { where } \quad a=\left(a, \frac{b+\sqrt{\Delta}}{2}\right) .
$$

It follows from (47) and (48) that for an integral ideal $b$ and some $a_{p} \geqslant$

$$
\text { (50) } \quad\left(a x_{0}+\frac{b+\sqrt{\Delta}}{2} y_{0}\right) \mathfrak{a}^{-1}=\mathfrak{b} \prod_{p=1}^{n} \frac{\left(\psi_{\nu}\left(\boldsymbol{t}_{2}\right)\right)^{\alpha_{p}}}{C\left(\psi_{p}\right)^{\alpha_{\nu}}}, \quad\left(\mathfrak{b}, \prod_{p=1}^{n} \frac{\left(\psi_{\nu}\left(\boldsymbol{t}_{2}\right)\right)}{O\left(\psi_{\nu}\right)}\right)=1 .
$$

On the other hand $\varphi_{v}^{e_{v}} \| f(\boldsymbol{t})$ implies $\phi_{v}^{\prime{ }_{p}} \| f(\boldsymbol{t})$, where $\varphi_{v}^{\prime}$ is conjugate to with respect to $\boldsymbol{Q}(\boldsymbol{t})$. If $\phi_{*} \notin \boldsymbol{Q}[\boldsymbol{t}]$ we have $\varphi_{\nu}^{\prime} \neq \phi_{p}$ and sinec $\phi_{n}^{\prime}$ hias t] coefficient of the leading term equal to 1 , by $(46)$

$$
\varphi_{\nu}^{\prime}=\varphi_{\lambda}, \quad e_{\nu}=e_{\lambda} ; \quad \psi_{n}^{\prime}=\psi_{\lambda} \quad \text { for } a, \lambda \neq \nu .
$$

Thus without loss of generality we may assume that for a certai $k \equiv n \bmod 2$

(51) $\quad \varphi_{\nu}^{\prime}=\varphi_{\nu^{\prime}}, \quad e_{p}=e_{y^{\prime}}, \quad \psi_{\nu}^{\prime}=\psi_{v^{\prime}}, \quad$ where $\quad \nu^{\prime}=\nu(1 \leqslant \nu \leqslant 7)$,

Hence by (48)

$$
v^{\prime}=\nu-(-1)^{n-\nu}(l<<v \leqslant n)
$$

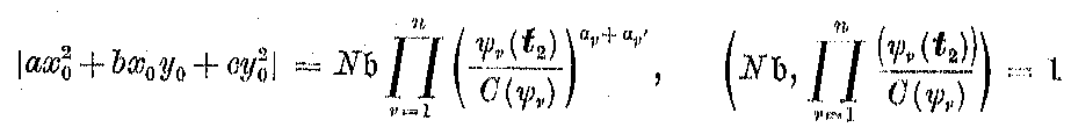

and a comparison with (49) gives

$$
a_{p}+a_{p^{\prime}}=e_{n} \quad(1 \leqslant \nu \leqslant n) .
$$

Let us define now $X(\boldsymbol{t}), Y(\boldsymbol{t})$ by the equation

(53) $\vartheta(\boldsymbol{t})=a X(\boldsymbol{t})+\frac{b+\sqrt{\Delta}}{2} X(\boldsymbol{t})=\left(a x_{0}+\frac{b+\sqrt{\Delta}}{2} y_{0}\right) \prod_{\text {pion } 1}^{n}\left(\frac{\varphi_{\nu}(\boldsymbol{t})}{\psi_{v}\left(\boldsymbol{t}_{2}\right)}\right)^{a_{\nu}}$.

The polynomials $X(\boldsymbol{t}), Y(\boldsymbol{t})$ have integral coefficients sinee by $(50)$

$$
\begin{aligned}
& C\{\eta(\mu \boldsymbol{t}+\tau)\}=\left(a x_{0}+\frac{b+\sqrt{\Delta}}{2} y_{0}\right) \prod_{v=1}^{n}\left(\frac{C\left(\psi_{v}\right)}{\left(\psi_{v}\left(\boldsymbol{t}_{2}\right)\right)}\right)^{a v}=\mathfrak{a} \mathfrak{b}, \\
& \mu^{|\vartheta|} \sigma(\vartheta) \equiv 0 \bmod \mathfrak{a}
\end{aligned}
$$

and $(\mu, a)=1$ implies $C(v) \equiv 0$ moda.

On the other hand, by $(53),(49),(51),(52),(46)$ and $(47)$

$$
\begin{aligned}
& F(X(\boldsymbol{t}), Y(\boldsymbol{t}))=a X(\boldsymbol{t})^{2}+b X(\boldsymbol{t}) Y(\boldsymbol{t})+c X(\boldsymbol{t})^{2}
\end{aligned}
$$

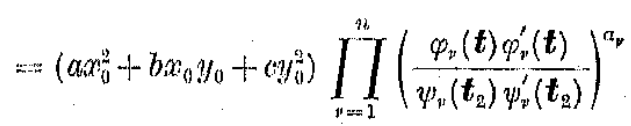

$$
\begin{aligned}
& =f\left(\mu \boldsymbol{t}_{2}+\tau\right) \prod_{v=1}^{n}\left(\frac{p_{v}(\boldsymbol{t})}{\psi_{\psi}\left(\boldsymbol{t}_{2}\right)}\right)^{a_{n}+a_{v^{\prime}}} \\
& =f\left(\mu \boldsymbol{t}_{2}+\tau\right) \prod_{p=1}^{n}\left(\frac{\varphi_{i}(\boldsymbol{t})}{\psi_{v}\left(\boldsymbol{t}_{2}\right)}\right)^{\gamma_{\nu}}=f(\boldsymbol{t}) .
\end{aligned}
$$

Assume now that $F$ is a reducible cubic form. If $F$ is singular we have $F^{T}=(a x+b y)^{2}(a x+a y)$, henee by the condition (1)

$$
\left|\begin{array}{ll}
a & b \\
a & d
\end{array}\right|= \pm 1
$$

If is equivalent to $x^{2} y$ and Theorem 1 applies.

If $F^{\prime}$ is non-singular we have

$$
\text { (54.) } \quad I^{\prime}(x, y)=\left(a_{0} x+b_{0} y\right) F_{1}(x, y),
$$

where $F_{1}$ is a non-singular primitive quadratic form. By Lemma 3 we have

$$
F_{1}(x, y)=G\left(a_{1} x+b_{1} y, a_{2} x+b_{2} y\right)
$$

where $G$ is primary and primitive. Let us put $G(x, y)=e x^{2}+g x y+h y^{2}$. By Lemma 2 , the discriminant $\Delta=g^{2}-4$ h equals 1 or is fundamental. The condition that $F$ is primary implies that

$$
d=\left(\begin{array}{ll}
a_{0} & a_{1} \\
b_{0} & b_{1}
\end{array}|,| \begin{array}{ll}
a_{1} & a_{2} \\
b_{1} & b_{2}
\end{array}|,| \begin{array}{ll}
a_{2} & a_{0} \\
b_{2} & b_{0}
\end{array} \mid\right)=1
$$

Otherwise, by a elcssical result on integral matrices (see [2], p. 52) the linear forms $a_{i} x+b_{i} y(0 \leqslant i \leqslant 2)$ would be expressible integrally in terms of two linear forms with determinant $d>1$. Let $\boldsymbol{K}=\boldsymbol{Q}(\sqrt{\Delta})$ and let the factorization of $f(\boldsymbol{t})$ over $\boldsymbol{K}$ be given by $(46)$. Sinee the fixed divisor of $f(\boldsymbol{t})$ equats $G(f)$ the condition (19) is sertisfied in virtne of Lemma 9 . By Lemma 6 the equation

(57)

$$
F(X(\boldsymbol{t}), X(\boldsymbol{t}))^{\circ}=f(\boldsymbol{t})
$$


has only finitely many solutions in polynomials $X(t), Y(t) \in Q[t]$ that are linearly independent. Let $M$ be a positive integer such that $M L X$, $M Y \in \boldsymbol{Z}[\boldsymbol{t}]$ for all of them. We apply Lemma 5 to the sequence $\left\{\varphi_{n}\right\}$ with this $M$. Let $\mu, \tau$ be any parameters with the property asserted in that Iemma and let $w_{v}(\boldsymbol{t})=\varphi_{v}(\boldsymbol{\mu} \boldsymbol{t}+\tau)$. We have again the formulae ( $\left.4 \tau\right)$ and $(48)$.

We shall deduce from $\mathbb{H}$ the existence of polynomials $\infty(\boldsymbol{t}), y(\boldsymbol{t}) \in \boldsymbol{Z}[\boldsymbol{t}]$ such that $F(x(t), y(\boldsymbol{t}))=f(\mu \boldsymbol{t}+\tau)$. This sulfices to prove the theorem. Indeed the polynomials

$$
X(\boldsymbol{t})=x\left(\frac{\boldsymbol{t}-\tau}{\mu}\right), \quad Y(\boldsymbol{t})=y\left(\frac{\boldsymbol{t}-\boldsymbol{\tau}}{\mu}\right)
$$

satisfy (57) and on one hand

$$
\mu^{|x|} \boldsymbol{X}, \mu^{|\nu|} Y \in \boldsymbol{Z}[\boldsymbol{t}]
$$

on the other hand if $X, X$ are linearly independent we have by the choice of $M$

$$
M X, M Y \in Z[t] .
$$

Since $(\mu, M)=1$ we get $X, Y \in \boldsymbol{Z}[\boldsymbol{t}]$.

If $X, Y$ are linearly dependent, then $F^{\prime}(X(\boldsymbol{t}), Y(\boldsymbol{t}))=f(\boldsymbol{t})$ $=C_{0}(f) f_{0}(t)^{3}, C\left(f_{0}\right)=1$ and

$$
\begin{gathered}
X(\boldsymbol{t})=\xi \zeta^{-1} f_{0}(\boldsymbol{t}), \quad \Psi(\boldsymbol{t})=\eta \zeta^{-1} f_{0}(\boldsymbol{t}), \quad \xi, \eta, \zeta \in Z,\langle\xi, \eta, \zeta)=1 \\
\quad H^{\prime}(\xi, \eta)=C(f) \zeta^{3}, \quad \zeta \mid \mu^{\left|f_{0}\right|} .
\end{gathered}
$$

If the above holds for all pairs $\left\langle\mu_{i}, \tau_{i}\right\rangle$ of the sequence mentioned in the last assertion of Xemma 5 then using the obvious notation we infer from $\left(\mu_{i}, \mu_{h}\right)=1$ that either $\left|\zeta_{i}\right| \neq\left|\zeta_{h}\right|$ for $i \neq h$ or there exists an $i$ with $\left|\zeta_{i}\right|=1$. In the former case since $\left|\zeta_{i}\right| \leqslant \mu_{i}^{\left|f_{0}\right|}$ the number of distinct $\left|\zeta_{i}\right| \leqslant Z$ is $\Omega\left(\frac{Z^{1 /\left|f_{0}\right| n}}{\log Z}\right)$, which contradicts Lemma 7. Therefore, the latter case holds and $X_{i}, Y_{i} \in Z[t]$.

In order to deduce the existence of $x(t), y(t)$ we slatl consider suceessively the cases $\Delta=1, \Delta<0, \Delta>1$.

If $\Delta=1$ by $(47) ;(48)$ and Lemma $5 \mathrm{II}$ implies the existence for every $\boldsymbol{t}_{1} \in Z^{r}$ and every $m$ prine to $f\left(\mu \boldsymbol{t}_{1}+\tau\right)$ of a $\boldsymbol{t}_{2}=\boldsymbol{t}_{1}$ mod $m$ such that $\frac{\left|\psi_{v}\left(\boldsymbol{t}_{2}\right)\right|}{O\left(\psi_{\nu}\right)}$ are distinct primes not dividing $B(1 \leqslant \nu \leqslant n)$.

On the other hand since a unimodular transformation of $G$ does not affect the condition (56) we can inssume $G(x, y)=x y$.

By the assumption of 0 there exist integers $x, y$ such that

$$
F(x, y)=f\left(\mu t_{2}+\tau\right)
$$

and it follows from $(47),(48),(54)$ and $(55)$ that for suitable integers $o_{i}$ and nonnegative integers $\alpha_{i,}(0 \leqslant i \leqslant 2,1 \leqslant \nu \leqslant n)$

$$
\begin{gathered}
a_{i} x+b_{i} y=o_{i} \prod_{\nu=1}^{n}\left(\frac{\psi_{\nu}\left(\boldsymbol{t}_{3}\right)}{C\left(\psi_{\nu}\right)}\right)^{a_{i v}}, \\
a_{0} c_{1} c_{2}=B_{k g n l} l, \quad a_{0 v}+\alpha_{1 v}+\alpha_{2 v}=e_{\nu} .
\end{gathered}
$$
that

$$
\prod_{s \in S} D_{s}\left(t_{2}\right)=0
$$

where for: $s=\left[\left\{a_{i}\right\},\left\{a_{i},\right\}\right]:$

$$
D_{s}(\boldsymbol{t})=\operatorname{act}\left[a_{i}, b_{i}, \Psi_{i s}(\boldsymbol{t})\right]_{0, i v i k 2}, \quad \Psi_{i s}(\boldsymbol{t})=c_{i} \prod_{y=1}^{n}\left(\frac{\psi_{y}(\boldsymbol{t})}{\sigma\left(\psi_{s}\right)}\right)^{a_{i n}}
$$

Since $\Psi_{i s}\left(\boldsymbol{t}_{2}\right) \equiv \Psi_{i s}\left(\boldsymbol{t}_{1}\right) \bmod m, D_{s}\left(\boldsymbol{t}_{2}\right) \equiv D_{s}\left(\boldsymbol{t}_{1}\right) \bmod m$ and $(60)$ gives

$$
\prod_{s \in S} D_{s}\left(t_{1}\right)=0 \bmod m \text {. }
$$

The latter eongruene holds for all $m$ prime to $f\left(\mu t_{1}+\tau\right)$, hence

$$
f\left(\mu t_{1}+\tau\right) \prod_{s \in S^{\prime}} D_{s}\left(t_{1}\right)=0
$$

and since $t_{1}$ is an arbitarary integral vector

$$
f(\mu t+\tau) \prod_{s \in S^{S}} D_{s}(t)=0
$$

identically. Ifowever $f(\mu t+\tau) \neq 0$, thus there exists an $s \in S$ such that

$$
D_{s}(\boldsymbol{t})=0
$$

By (56) the rank of the matrix $\left[a_{i}, b_{i}\right]_{0 \leqslant i \leqslant 2}$ is two, thus the system of equations

$$
a_{i} x+b_{i} y=\Psi_{i s}(t) \quad(0 \leqslant i \leqslant 2)
$$

is soltublo in polynomials $x, y \in Q[t]$.

Moreover, by Cramer's formulat

$$
\left|\begin{array}{ll}
a_{i} & b_{i} \\
a_{j} & b_{j}
\end{array}\right| x,\left|\begin{array}{ll}
a_{i} & b_{i} \\
a_{j} & b_{j}
\end{array}\right| y \in \boldsymbol{Z}[\boldsymbol{t}] \quad(0 \leqslant i \leqslant j \leqslant 2)
$$

and agatia by $(56) x, y \in Z[t]$. On the other hand by $(59),(48)$ and $(47)$

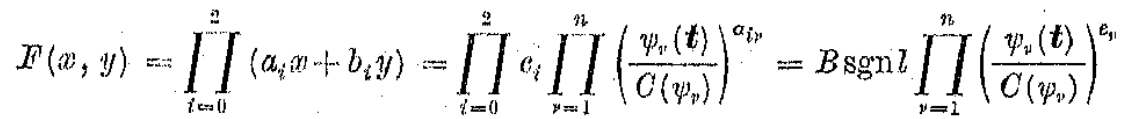

$$
\begin{aligned}
& =l \prod_{p=1}^{n} \psi_{p}(\boldsymbol{t})^{e_{n}}=f(\mu \boldsymbol{t}+\tau) \text {. }
\end{aligned}
$$


Tet ins consiger now the case $A \neq 1$. Then by Lemma 5 and $(4: 7),(48) \mathrm{H}$ implies the existence for every $t_{1} \in Z^{r}$ and every $m$ prime to $\Delta f\left(\mu t_{1}+\tau\right)$ of a $\boldsymbol{t}_{2} \equiv \boldsymbol{t}_{1} \bmod m$ such that the ideals $\frac{\left(\psi_{\eta}\left(\boldsymbol{t}_{2}\right) \mid\right.}{C\left(\psi_{v}\right)}(\nu \leqslant n)$ are prime in $\boldsymbol{K}$, distinct and do not divide $B$. By the assumption of $\mathrm{O}$ there exist integers $x, y$ such that

$$
F(x, y)=f\left(\mu t_{2}+\tau\right)
$$

and it follows from (47), (48), (51) and (55) that for suitable integral ideals $a, b$ and nonnegative integers $\alpha_{v}, \beta_{v},(1 \leqslant v \leqslant n)$

$$
\left(a_{0} x+b_{0} y\right)=\mathfrak{a} \prod_{v=1}^{n}\left(\frac{\left(\psi_{y}\left(\boldsymbol{t}_{2}\right)\right]}{C\left(\psi_{v}\right)}\right)^{\mathfrak{a}_{p}},
$$

$$
\left(e\left(a_{1} x+b_{1} y\right)+\frac{y+\sqrt{\Delta}}{2}\left(a_{2}\left(x+b_{2} y\right)\right) \mathfrak{g}^{-1}=\mathfrak{b} \prod_{v=1}^{b}\left(\frac{\psi_{y}\left(t_{2}\right)}{O\left(\psi_{v}\right)}\right)^{\beta_{v}}\right.
$$

where $g=\left(e, \frac{g+\sqrt{\Delta}}{2}\right)$,

(62) $\quad \mathfrak{a} N \mathfrak{B}=(B), \quad \alpha_{v}+\beta_{v}+\beta_{w^{\prime}}=c_{v} \quad(1 \leqslant \nu \leqslant n)$.

We get

$$
a_{0} x+b_{0} y=a \prod_{y=1}^{n} \psi_{y}\left(\boldsymbol{t}_{2}\right)^{\alpha_{n}}
$$

$$
e\left(a_{1} x+b_{1} y\right)+\frac{g+\sqrt{4}}{2}\left(a_{2} x+b_{2} y\right)=\beta \prod_{p=1}^{n} \psi_{v}\left(t_{1}\right)^{\beta_{r}}
$$

where

$$
(\alpha)=\mathfrak{a} \prod_{n=1}^{n} O\left(\psi_{w}\right)^{-a_{n}}, \quad(\beta)=\mathfrak{g} \mathfrak{b} \prod_{v=1}^{n} O\left(\psi_{v}\right)^{-\beta_{v}}
$$

and by (47) and (62)

(65)

$$
\alpha N \beta=l e
$$

Since $\mathfrak{a}$ is integral $\Psi_{0}\left(t ; \alpha, \alpha_{p}\right)=\alpha \prod_{p=1}^{n} \psi_{p}(t)^{\alpha_{p}}$ lias integral coefficiontis. On the other hand, by (51) and (62) $a_{y}=\alpha_{k^{\prime}}(k<y \leqslant n)$ and by $(65)$ $a \in Q$, hence

$$
\Psi_{0}\left(\boldsymbol{t} ; \alpha, \alpha_{y}\right) \in \boldsymbol{Z}[\boldsymbol{t}]
$$

Similarly, since $\mathfrak{b}$ is integral $\beta \prod_{r=1}^{n} \psi_{v}(\boldsymbol{t})^{\beta_{n}} \in \mathfrak{g}[\boldsymbol{t}]$ and we get

$$
\beta \prod_{v=1}^{n} \psi_{v}(\boldsymbol{t})^{\beta_{v}}=e \Psi_{1}\left(\boldsymbol{t} ; \beta, \beta_{v}\right)+\frac{g+-\sqrt{\Lambda}}{2} \Psi_{2}\left(\boldsymbol{t} ; \beta, \beta_{p}\right),
$$

where

$$
\Psi_{i}\left(\boldsymbol{t} ; \beta, \beta_{v}\right) \in \boldsymbol{Z}[\boldsymbol{t}] \quad(i=1,2) .
$$

The equations (63) take the form

$$
\begin{gathered}
a_{0} x+b_{0} y=\Psi_{0}\left(\boldsymbol{t}_{2} ; \alpha, \alpha_{\nu}\right) \\
a_{i} x+b_{i} y=\Psi_{i}\left(\boldsymbol{t}_{2} ; \beta, \beta_{,}\right) \quad(i=1,2) .
\end{gathered}
$$

For a system $s=\left[\alpha, \beta,\left\{\alpha_{v}\right\},\left\{\beta_{i}\right\}\right]$ put

$$
\Psi_{0 s}(\boldsymbol{t})=\Psi_{0}\left(\boldsymbol{t} ; \alpha, \alpha_{v}\right), \quad \Psi_{i, s}(\boldsymbol{t})=\Psi_{i}\left(\boldsymbol{t} ; \beta, \beta_{i}\right) \quad(i=1,2)
$$

and denote by $S$ the set of all such systems satisfying (62) and (64). If $\Delta<0$ the set $S$ is finite. It follows from $(67)$ that

$$
\prod_{S \in S} D_{s}\left(\boldsymbol{t}_{2}\right)=0
$$

where

$$
D_{s}(\boldsymbol{t})=\operatorname{det}\left[a_{i}, b_{i}, \Psi_{i s}(\boldsymbol{t})\right]_{0 \leqslant i \leqslant 2} .
$$

Since $\Psi_{i s}\left(\boldsymbol{t}_{2}\right) \equiv \Psi_{i s}\left(\boldsymbol{t}_{1}\right) \bmod m$ we infer from $(68)$ as in the case $\Delta=1$ from $(60)$ that for a suitable $s \in S$ the system of equations

$$
a_{i} x+b_{i} y=\Psi_{i s}(\boldsymbol{t}) \quad(0 \leqslant i \leqslant 2)
$$

is soluble in polynomials $x, y \in \boldsymbol{Z}[\boldsymbol{t}]$. By $(54),(55),(66),(47),(65)$ and (51) we get

$$
\begin{aligned}
& F^{\prime}(x, y)=\left(a_{0} x+b_{0} y\right) N\left(e\left(a_{1} x+b_{1} y\right)+\frac{g+\sqrt{\Delta}}{2}\left(a_{2} x+b_{2} y\right)\right) e^{-1} \\
& =\Psi_{0 s}(\boldsymbol{t}) N\left(e \Psi_{1 s}(\boldsymbol{t})+\frac{g+\sqrt{\Delta}}{2} \Psi_{2 s}(\boldsymbol{t})\right) e^{-1} \\
& =\alpha \prod_{\nu=1}^{n} \psi_{\nu}(\boldsymbol{t})^{\alpha_{\nu}} N\left(\beta \prod_{\nu=1}^{n} \psi_{\nu}(\boldsymbol{t})^{\beta_{\nu}}\right) e^{-1}
\end{aligned}
$$

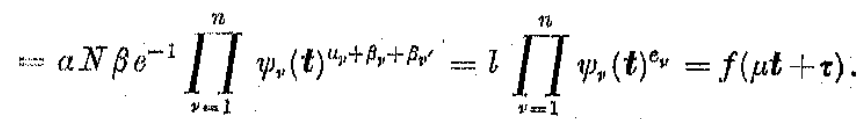

If $\Delta>0$ the set $S$ is infinite. Wo can however divide it into finitely many classes assigning two systems $\left[\alpha, \beta,\left\{\alpha_{\psi}\right\},\left\{\beta_{\nu}\right\}\right]$ and $\left[\alpha, \gamma,\left\{\alpha_{\nu}\right\},\left\{\beta_{\nu}\right\}\right]$ to the same class if $\gamma / \beta$ is a totally positive unit of $\boldsymbol{K}$. Then every class contains exactly one system satisfying

$$
1 \leqslant|\beta|<\varepsilon,
$$

where $\varepsilon>1$ is the fnndamental totally positive unit. Denoting the set of all systems satisfying $(62),(64)$ and $(68)$ by $\mathbb{S}_{0}$ we infer from $(67)$ the 
existence of a $\sigma \in \boldsymbol{Z}$ such that

$$
\prod_{s \in S_{0}} D_{\sigma s}\left(\boldsymbol{t}_{2}\right)=0
$$

where for $s=\left[\alpha, \beta,\left\{\alpha_{\psi}\right\},\left\{\beta_{v}\right\}\right]$

$$
D_{\sigma s}(\boldsymbol{t})=\left|\begin{array}{lll}
a_{0} & b_{0} & \Psi_{0}\left(\boldsymbol{t} ; \alpha, \alpha_{v}\right) \\
a_{1} & b_{1} & \Psi_{1}\left(\boldsymbol{t} ; \varepsilon^{\sigma} \beta, \beta_{v}\right) \\
a_{2} & b_{2} & \Psi_{2}\left(\boldsymbol{t} ; \varepsilon^{\sigma} \beta, \beta_{v}\right)
\end{array}\right|
$$

Since $D_{\sigma s}\left(\boldsymbol{t}_{2}\right) \equiv D_{\sigma s}\left(\boldsymbol{t}_{1}\right) \bmod m$ for all $s$ we conclude that

$$
\prod_{s \in S_{0}} D_{\sigma s}\left(\boldsymbol{t}_{1}\right)=0 \bmod m
$$

where $\sigma$ depends on $m$.

We liave an identity

$$
u\left(e \Psi_{18}(\boldsymbol{t})+\frac{g+\sqrt{\Delta}}{2} \Psi_{2 s}(\boldsymbol{t})\right)=e \Phi_{1 s}(\boldsymbol{t}, u)+\frac{g+\sqrt{\Delta}}{2} \Phi_{2 s}(\boldsymbol{t}, u)
$$

where

$$
\begin{array}{r}
\Phi_{1 s}(\boldsymbol{t}, u)=\frac{1}{2}\left[u\left(1-\frac{g}{\sqrt{\Delta}}\right)+u^{-1}\left(1+\frac{g}{\sqrt{\Delta}}\right)\right] \Psi_{1 s}(\boldsymbol{t})-h \frac{u-u^{-1}}{\sqrt{\Delta}} \Psi_{2 s}^{\prime}(\boldsymbol{t}) \\
\Phi_{2 s}(\boldsymbol{t}, u)=e \frac{u-u^{-1}}{\sqrt{\Delta}} \Psi_{i s}(\boldsymbol{t})+\frac{1}{2}\left[u\left(1-\frac{g}{\sqrt{\Delta}}\right)+u^{-1}\left(1+\frac{g}{\sqrt{\Delta}}\right)\right] \Psi_{2 s}(\boldsymbol{t})+ \\
+g \frac{u-u^{-1}}{\sqrt{\Delta}} \Psi_{2 s}(\boldsymbol{t})
\end{array}
$$

Since $\varepsilon$ is conjugate to $\varepsilon^{-1}$

$$
\Phi_{i s}\left(\boldsymbol{t}, \varepsilon^{\sigma}\right) \in \boldsymbol{Q}[\boldsymbol{t}] \quad(i=1,2)
$$

and by (71)

$$
\Psi_{i}\left(\boldsymbol{t} ; \varepsilon^{\sigma} \beta, \beta_{v}\right)=\Phi_{i s}\left(\boldsymbol{t}, \varepsilon^{\sigma}\right) \quad \cdots(i=1,2)
$$

The congruence (70) takes the form

$$
\prod_{s \in S_{0}} E_{s}\left(\boldsymbol{t}_{1}, \varepsilon^{\sigma}\right)=0 \bmod m
$$

where

$$
E_{s}(\boldsymbol{t}, u)=\left|\begin{array}{lll}
a_{0} & b_{0} & \Psi_{0 s}(\boldsymbol{t}) \\
a_{1} & b_{1} & \Phi_{1 s}(\boldsymbol{t}, u) \\
a_{2} & b_{2} & \Phi_{2 s}(\boldsymbol{t}, u)
\end{array}\right|
$$

However $u E(\boldsymbol{t}, u) \in \boldsymbol{Q}[\boldsymbol{t}, u]$ and hence $u^{\left|S_{0}\right|} \prod_{\boldsymbol{\delta} \in S_{0}} E\left(\boldsymbol{t}_{1}, u\right) \in \boldsymbol{Q}[u]$.
Since the congruence (72) is soluble for all $m$ prime to $\Delta f\left(\mu \boldsymbol{t}_{1}+\tau\right)$ it follows from Theorem 6 of [15] that the equation.

$$
f\left(\mu \boldsymbol{t}_{1}+\tau\right) \prod_{s \in S_{0}} E_{s}\left(\boldsymbol{t}_{1}, \varepsilon^{\sigma}\right)=0
$$

is soluble in integers $\sigma$. Thus for every $\boldsymbol{t}_{1} \in \boldsymbol{Z}^{r}$ either $f\left(\mu \boldsymbol{t}_{1}+\tau\right)=0$ or $f\left(\mu \boldsymbol{t}_{1}+\tau\right) \neq 0$ and there exist $a \quad \sigma \in Z$ and an $s=\left[\alpha, \beta,\left\{\alpha_{v}\right\},\left\{\beta_{v}\right\}\right] \in S_{0}$
such that $W_{s}\left(\boldsymbol{t}_{1}, \varepsilon^{\sigma}\right)=0$.

In the latter case it follows from (71) and (73) that

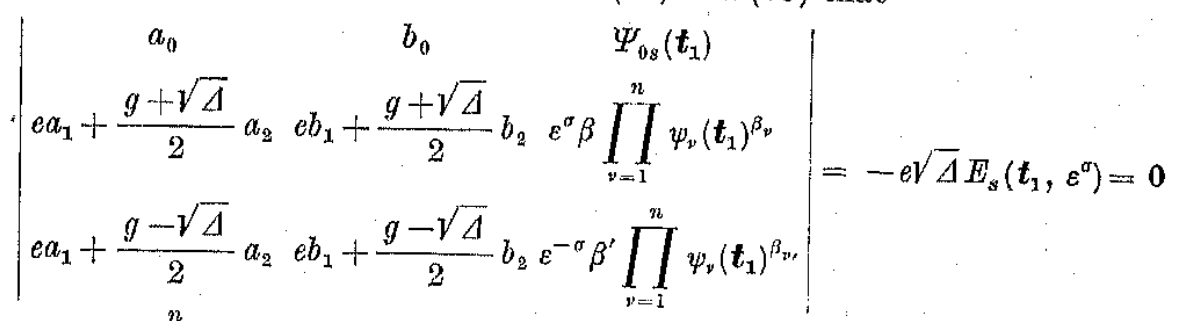
and $\varepsilon^{\alpha} \beta \prod_{\nu=1}^{n} \psi_{\nu}\left(\boldsymbol{t}_{1}\right)^{\beta_{*}}$ satisfies the quadratic equation

$$
L z^{2}-\boldsymbol{K} \Psi_{0 s}\left(\boldsymbol{t}_{1}\right) z-L^{\prime} N \beta \prod_{\nu=1}^{n} \psi_{\nu}\left(\boldsymbol{t}_{1}\right)^{\beta_{\nu}+\beta_{y^{\prime}}}=0,
$$

where $\beta^{\prime}, L^{\prime}$ are conjugate to $\beta, L$ respcetively

$$
\begin{aligned}
& L=\left|\begin{array}{cc}
a_{0} & b_{0} \\
e a_{1}+\frac{g-\sqrt{\Delta}}{2} a_{2} & e b_{1}+\frac{g-\sqrt{\Delta}}{2} b_{2}
\end{array}\right|, \\
& K=\left|\begin{array}{cc}
e a_{1}+\frac{g+\sqrt{\Lambda}}{2} a_{2} & e b_{1}+\frac{g+\sqrt{\Delta}}{2} b_{2} \\
e a_{1}+\frac{g-\sqrt{\Delta}}{2} a_{2} & e b_{1}+\frac{g-\sqrt{\Delta}}{2} b_{2}
\end{array}\right| .
\end{aligned}
$$

Since $e\left[a_{0}, b_{0}\right] \neq 0$ we bare $L \neq 0$ by $(56)$, and

$$
\left|\varepsilon^{\sigma} \beta \prod_{\nu=1}^{n} \psi_{\nu}\left(\boldsymbol{t}_{1}\right)^{\beta_{\nu}}\right| \ll\left\|\boldsymbol{t}_{1}\right\|^{|f|}
$$

where 7 denotes the maximum modulus of the conjugates and the constant in the symbol $\ll$ depends on $F, f, \mu, \tau, s$.

On the other hand, by $(47),(51),(62),(65)$ and $(69)$

$$
\beta \prod_{\nu=1}^{n} \psi_{\nu}\left(\boldsymbol{t}_{1}\right)^{\beta_{v}} \mid \ll\left\|\boldsymbol{t}_{1}\right\|^{f f / 2}
$$


Since $f\left(\mu t_{1}+\tau\right) \neq 0$ whence by $(64)$

$$
\left|N\left(\beta \prod_{p=1}^{n} \psi_{\nu}\left(\boldsymbol{t}_{1}\right)^{\beta_{\nu}}\right)\right| \gg N \mathrm{gb} \gg 1
$$

we get

$$
\left|\beta^{-1} \prod_{\nu=1}^{n} \psi_{\nu}\left(\boldsymbol{t}_{1}\right)^{-\beta_{v}}\right|<\left\|\boldsymbol{t}_{1}\right\|^{|f| / 2}
$$

This together with (75) implies

$$
\varepsilon^{|\sigma|}=\sqrt{\varepsilon^{\sigma} \mid} \leqslant\left\|\boldsymbol{t}_{1}\right\|^{\frac{3}{2}|f|}, \quad|\sigma| \leqslant \frac{3}{2}|f| \frac{\log || \boldsymbol{t}_{1} \|}{\log \varepsilon}+\varrho
$$

where $\varrho$ is a constant depending on $F, f, \mu, \tau$ but independent of $s$ $\left(S_{0}\right.$ is finite).

Let us choose now a positive integer $T$ so large that

$$
2 T+1>|f|\left(\left|S_{0}\right|+1\right)\left(3|f| \frac{\log T}{\log \varepsilon}+2 \varrho+1\right)
$$

If $\boldsymbol{t}_{1}$ runs through all integral vectors satisfying $\left\|\boldsymbol{t}_{1}\right\| \leqslant T \sigma$ runs through integers satisfying

$$
|\sigma| \leqslant \frac{3}{2}|f| \frac{\log T}{\log \varepsilon}+\varrho
$$

The number of vectors in question is $(2 T+1)^{r}$, the number of integers does not exceed $3|f| \frac{\log T}{\log \varepsilon}+2 \varrho+1$, hence there is an integer $\sigma_{0}$ that corresponds to at least

$$
(2 T+1)^{r}\left(3|f| \frac{\log T}{\log \varepsilon}+2 \varrho+1\right)^{-1}
$$

different vectors $\boldsymbol{t}_{1}$ satistying $\left\|\boldsymbol{t}_{1}\right\| \leqslant T . B y(76)$ we get more than $|f|\left(\left|S_{0}\right|+1\right) \times$ $\times(2 T+1)^{r-1}$ such vectors satisfying the equation

$$
f\left(\mu t_{1}+\tau\right) \prod_{s \in S_{0}} E_{s}\left(t_{1}, \varepsilon^{\sigma_{0}}\right)=0 .
$$

Since by $(62),(71)$ and $(73)$ the degree of $D_{s}\left(t, \varepsilon^{\sigma_{0}}\right)$ does not exceed $|f|$ the degxee of the polynomial on the left-hand side does not exceed $|f|\left(\left|S_{0}\right|+1\right)$ and Lemma 8 shows that

$$
f(\mu \boldsymbol{t}+\tau) \prod_{s \in S_{0}} E_{s}\left(\boldsymbol{t}, \varepsilon^{\sigma_{0}}\right)=0
$$

identically. Therefore, there exists an $s \in S_{0}$ such that $E_{s}\left(\boldsymbol{t}, \varepsilon^{\sigma_{0}}\right)=0$ and by (56) the system of equations

$$
\begin{aligned}
& a_{0} x+b_{0} y=\Psi_{0 s}(\boldsymbol{t}), \\
& a_{i} x+b_{i} y=\Phi_{i s}(\boldsymbol{t}) \quad(i=1,2)
\end{aligned}
$$

is soluble in polynomials $x, y \in \boldsymbol{Z}[\boldsymbol{t}]$. By $(54),(55),(71),(66),(47),(62),(65)$ and (51) we get for these polynomials

$$
\begin{aligned}
F(x, y) & =\left(a_{0} x+b_{0} y\right) N\left(e\left(a_{1} x+b_{1} y\right)+\frac{g+\sqrt{\Delta}}{2}\left(a_{2} x+b_{2} y\right)\right) e^{-1} \\
& =\Psi_{0 s}(\boldsymbol{t}) N\left(\varepsilon^{-\sigma_{0}} e\left(a_{1} x+b_{1} y\right)+\varepsilon^{-\sigma_{0}} \frac{g+\sqrt{\Delta}}{2}\left(a_{2} x+b_{2} y\right)\right) e^{-1} \\
& =\Psi_{0 s}(\boldsymbol{t}) N\left(e \Psi_{i s}(\boldsymbol{t})+\frac{g+\sqrt{\Delta}}{2} \Psi_{2 s}(\boldsymbol{t})\right) e^{-1}=f(\boldsymbol{\mu} \boldsymbol{t}+\tau)
\end{aligned}
$$

and the proof is complete.

Remark. For the proof of a more general result mentioned in the introduction one needs more general versions of Lemmata 2,5 and 7 and Theorem 7 of [16] instead of Theorem 6 of [15]. In the difficult case of an irreducible form $F$ with all zeros real Theorem 7 of [16] does not suffice, but Skolem's conjecture on exponential congruences would do (see [18]). One could avoid this step in the proof provided it were known that the number of vectors $t$ satisfying $\|t\| \leqslant T$ and the conditions of Lemma 4 grows faster than $T^{r-1}(\log T)^{|F|}$. For $r=1$ much more has been conjectured by Bateman and Horn [1].

4. The next lemma is a refinement of Lemma 1 of [13].

LAMMa 10. Let $P \in \boldsymbol{Q}[\boldsymbol{t}, u]$ be a polynomial swoh that for no $\varphi \in \boldsymbol{Q}(\boldsymbol{t})$

$$
P(\boldsymbol{t}, p(\boldsymbol{t}))=0
$$

identioally. Then there exists a $\boldsymbol{t}_{1} \in Z^{r}$ such that for any $M \in \boldsymbol{N}$ there exists an $m \in \boldsymbol{N}$ prime to $M$ such that for all $\boldsymbol{t} \in \boldsymbol{Z}, \boldsymbol{t} \equiv \boldsymbol{t}_{1} \bmod m$ and all $u \in \boldsymbol{Q}$

$$
P(t, u) \neq 0 \text {. }
$$

Proof. Following the proof of Lemma 1 in $[13]$ we take $m=q_{1} \ldots q_{k}$, where in the notation of that paper the primes $q_{i}$ are chosen not to divide $M$.

LxMvia 11. Let $G, H \in Q[x, y]$ be relatively prime forms, $p, g_{i}, h_{i} \in Q[t]$ $(i \leqslant I)$ arbitrary polynomials, $p \neq 0$.

If for every $\boldsymbol{t}_{1} \in \boldsymbol{Z}^{r}$ and for every integer $m$ prime to $p(\boldsymbol{t})$ there are an $i \leqslant I$, a $\boldsymbol{t}_{2} \in \boldsymbol{Z}^{r}, \boldsymbol{t}_{2}=\boldsymbol{t}_{1} \bmod m$ and $x, y \in \boldsymbol{Q}$ satisfying

$$
G(x, y)=g_{i}\left(\boldsymbol{t}_{2}\right), \quad H(x, y)=h_{i}\left(\boldsymbol{t}_{2}\right)
$$

then there exist $a j \leqslant I$ and polynomials $X, X \in Q[\boldsymbol{t}]$ such that

$$
G(X, Y)=g_{j}, \quad H(X, Y)=h_{j} .
$$


Proof. If $G(x, y)-g_{i}(t), H(x, y)-h_{i}(\boldsymbol{t})$ had a common factor $d(x, y, t) \neq$ const then the leading forms of $d$ with respect to $x, y$ would divide $G(x, y)$ and $H(x, y)$. Thus for each $i \leqslant I$

$$
\left\{G(x, y)-g_{i}(\boldsymbol{t}), H(x, y)-h_{i}(\boldsymbol{t})\right)=1 \text {. }
$$

Let $R_{i}(t, x), S_{i}(t, y)$ be the resultants of $G(x, y)-g_{i}(\boldsymbol{t})$ and $H(x, y)-h_{i}(\boldsymbol{t})$ with respect to $y$ and $x$ respectively. It follows from the construction of resultants that the leading coefficients of $R_{i}$ in $x$ and of $S_{i}$ in $y$ are equal to the resultants of $G(1, z), H(1, z)$ and of $G(z, 1), H(z, 1)$ respectively. Hence these leading coefficients are independent of $t$. Let

$$
\begin{aligned}
& R_{i}(\boldsymbol{t}, x)=R_{i 0}(\boldsymbol{t}, x) \prod_{\substack{\ell=1 \\
s_{i}}}^{r_{i}}\left(x-R_{i \varrho}(\boldsymbol{t})\right), \\
& S_{i}(\boldsymbol{t}, y)=S_{i 0}(\boldsymbol{t}, y) \prod_{\sigma=1}^{s_{\sigma}}\left(y-S_{i \sigma}(\boldsymbol{t})\right)
\end{aligned}
$$

where $R_{i 0}$ and $S_{i 0}$ have no factor linear in $x$ or $y$ respectively. Tf for some triple $(i, \varrho, \sigma)$ with $i \leqslant I, 1 \leqslant \varrho \leqslant r_{i}, 1 \leqslant \sigma \leqslant s_{j}$

$$
G\left(R_{i \varrho}, S_{i \sigma}\right)=g_{i} \quad \text { and } \quad H\left(R_{i \varrho}, S_{i \sigma}\right)=h_{i}
$$

the lemma follows.

Therefore, suppose that for each triple $(i, \varrho, \sigma)$ in question

Then

$$
G\left(R_{i 0}, S_{i \sigma}\right) \neq g_{i} \quad \text { or } \quad H\left(R_{i 0}, S_{i \sigma}\right) \neq h_{i} .
$$

$$
T_{i \varrho \sigma}=\left\langle\left(G\left(R_{i \sigma}, S_{i \sigma}\right)-g_{i}\right)^{2}+\left(H\left(R_{i \varrho}, S_{i \sigma}\right)-h_{i}\right)^{2} \neq 0\right.
$$

and we set in Lemma 10

$$
P(\boldsymbol{t}, u)=p(\boldsymbol{t}) \prod_{i=1}^{I} R_{i 0}(\boldsymbol{t}, u) S_{i 0}(\boldsymbol{t}, u) \prod_{\ell=1}^{r_{i}} \prod_{\sigma=1}^{s_{i}} T_{i \varrho \sigma}(\boldsymbol{t})
$$

By that lemma with $M=1$ there exist an $m \in \boldsymbol{N}$ and a $\boldsymbol{t}_{1} \in \boldsymbol{Z}^{r}$ such that if $\boldsymbol{t} \equiv \boldsymbol{t}_{1} \bmod m$ and $u \in \boldsymbol{Q}$ we have

$$
P(\boldsymbol{t}, u) \neq 0
$$

In particular, taking $\boldsymbol{t}=\boldsymbol{t}_{1}$ we get $p\left(\boldsymbol{t}_{1}\right) \neq 0$. Applying Lemma 10 again with $M=p\left(t_{1}\right)$ we infer the existence of an integer $m$ with the above property satisfying $\left(m, p\left(t_{1}\right)\right)=1$. However now by the assumption there exists an $i \leqslant I$, a $\boldsymbol{t}_{2}=\boldsymbol{t}_{1} \bmod m$ and $x, y \in Q$ such that (77) holds. By the fundamental property of resultants we have

$$
R_{i}(t, x)=0=S_{i}(t, y)
$$

and in view of $(78),(79),(81)$ and $(82)$ there exist $\varrho, \sigma$ such that $1 \leqslant \varrho \leqslant r_{i}$, $1 \leqslant \sigma \leqslant s_{i}$

$$
x=R_{i_{R}}\left(t_{2}\right), \quad y=S_{i \sigma}\left(t_{2}\right)
$$

It follows from (77) and (80) that

$$
T_{i \varrho \sigma}\left(\boldsymbol{t}_{2}\right)=0,
$$

contrary to (81) and (82).

Remark. Lemma 11 extends to any system of forms $G_{1}, G_{2}, \ldots, G_{k}$ $\in \boldsymbol{Q}\left[x_{1}, \ldots, x_{k}\right]$ without a common non-trivial zero.

Proof of Theorem 3. If $f=0$ the theorem is trivially true. If $f \neq 0$ let $f\left(t_{0}\right)=e \neq 0$. We set $f_{0}(\boldsymbol{t})=f\left(e t+t_{0}\right)$ and find as in the proof of Corollary to Lemma 3 that the fixed divisor of $f_{0}(t)$ equals $O\left(f_{0}\right)$. (If the fixed divisor of $f$ equals $O(f)$ we can take directly $e=1, \boldsymbol{t}_{0}=\mathbf{0}$.) Let $\boldsymbol{K}$ be the least field over which $F$ factorizes into two coprime factors and let

$$
f_{0}(\boldsymbol{t})=l \prod_{\nu=1}^{n} \varphi_{\nu}(\boldsymbol{t})^{e_{\nu}}
$$

be a factorization of $f$ over $\boldsymbol{K}$ into irreducible factors such that $\phi_{\nu}$ are distinct and have the coefficient of the first teim in the antilexicographic order equal to 1 . Since the fixed divisor of $f_{0}(\boldsymbol{t})$ equals $G\left(f_{0}\right)$ the polynomials $\varphi_{v}$ satisfy (19) in virtue of Lemma 9 . Let $\mu, \tau$ be parameters whose existence for $\left\{\varphi_{\nu}\right\}$ and $\mu=1$ is asserted in Lemma 5 and let.

$$
\psi_{\nu}=p_{v}(\mu \boldsymbol{t}+\boldsymbol{r}) \quad(1 \leqslant \nu \leqslant n) .
$$

It follows that

$$
f_{0}(\mu \boldsymbol{t}+\boldsymbol{\tau})=l \prod_{\nu=1}^{n} \psi_{\nu}(\boldsymbol{t})^{c_{\nu}}
$$

and

$$
B=|l| \prod_{\nu=1}^{n} O\left(\psi_{\nu}\right)^{e_{\eta}}=O\left(f_{0}(\mu \boldsymbol{t}+\boldsymbol{\tau})\right) \in \boldsymbol{N}
$$

where an ideal in $Q$ is identified with its positive generator: Consider first the case where $\boldsymbol{K}=\boldsymbol{Q}$ and let

$$
f_{0}(\mu \boldsymbol{t}+\boldsymbol{x})=g_{i}(\boldsymbol{t}) h_{i}(\boldsymbol{t}) \quad(1, \leqslant i \leqslant I)
$$

be all possible factorizations of the loft-hand side into two factors with integral coefficients. If implies that if $\left(m, f_{0}\left(\mu \boldsymbol{t}_{1}+\boldsymbol{x}\right)\right)=1$ there exist an $i \leqslant I$, a $t_{2}=t_{1}$ mod $m$ and $x, y \in Z$ such that

$$
Q(x, y)=g_{i}\left(t_{2}\right) ; \quad H(x, y)=h_{i}\left(t_{2}\right) .
$$

Indeed, by (84) and (85) the condition $\left(m, f_{0}\left(\mu \boldsymbol{t}_{1}+\tau\right)\right)=1$ implies

$$
\left(m, \prod_{\nu=1}^{n} \frac{\psi_{\nu}\left(\boldsymbol{t}_{1}\right)}{O\left(\psi_{\nu}\right)}\right)=1
$$


and by Lemma 5 Himplies the existence of a $\boldsymbol{t}_{2} \in \boldsymbol{Z}^{r}, \boldsymbol{t}_{2} \equiv \boldsymbol{t}_{1} \bmod m$ such that $\frac{\left|\psi_{\nu}\left(\boldsymbol{t}_{2}\right)\right|}{O\left(\psi_{\nu}\right)}(\nu \leqslant n)$ are distinct primes not dividing $B$. By the assumption of $\mathbf{D}$ there exist $x, y \in \boldsymbol{Z}$ such that

$$
G(x, y) H(x, y)=F(x, y)=f_{0}\left(\mu t_{2}+\tau\right)
$$

and it follows from (84) and (85) that for some $a, b, \alpha_{v}, \beta_{v} \in Z, a_{v} \geqslant 0$, $\beta_{y} \geqslant 0$ we have

$$
\begin{gathered}
G(x, y)=a \prod_{\nu=1}^{n}\left(\frac{\psi_{\nu}\left(\boldsymbol{t}_{2}\right)}{O\left(\psi_{\nu}\right)}\right)^{a_{\nu}}, \quad H(x, y)=b \prod_{\nu=1}^{n}\left(\frac{\psi_{\nu}\left(\boldsymbol{t}_{2}\right)}{O\left(\psi_{\nu}\right)}\right)^{\beta_{\nu},}, \\
a b=B \operatorname{sgn} l, \quad \alpha_{\nu}+\beta_{\nu}=e_{\nu} \quad(1 \leqslant \nu \leqslant n) .
\end{gathered}
$$

Taking

$$
g_{i}(t)=a \prod_{\nu=1}^{n}\left(\frac{\psi_{\nu}(\boldsymbol{t})}{C\left(\psi_{\nu}\right)}\right)^{\alpha_{\nu}}, \quad \hbar_{i}(t)=b \prod_{\nu=1}^{n}\left(\frac{\psi_{\nu}(\boldsymbol{t})}{C\left(\psi_{v}\right)}\right)^{\beta_{\nu}}
$$

we get (86) and (87). Now we apply Lemma 11 with $p(\boldsymbol{t})=f_{0}(\mu \boldsymbol{t}+\tau)$ and we get the existence of $X_{0}, X_{0} \in Q[t]$ satisfying

$$
G\left(X_{0}, Y_{0}\right)=g_{j}, \quad H\left(X_{0}, Y_{0}\right)=h_{j}
$$

for some $j \leqslant I$. Setting

we get by $(86)$

$$
X(\boldsymbol{t})=X_{0}\left(\frac{\boldsymbol{t}-e \tau-\boldsymbol{t}_{0}}{e \mu}\right), \quad Y(\boldsymbol{t})=Y_{0}\left(\frac{\boldsymbol{t}-e \tau-\boldsymbol{t}_{0}}{e \mu}\right)
$$

$$
F(X(\boldsymbol{t}), Y(\boldsymbol{t}))=g_{j}\left(\frac{\boldsymbol{t}-e \boldsymbol{\tau}-\boldsymbol{t}_{0}}{e \mu}\right) h_{j}\left(\frac{\boldsymbol{t}-e \boldsymbol{\tau}-\boldsymbol{t}_{0}}{e \mu}\right)=f_{0}\left(\frac{\boldsymbol{t}-\boldsymbol{t}_{0}}{e}\right)=f(\boldsymbol{t}) .
$$

Consider now the case where $\boldsymbol{K}$ is an imaginary quadratic field with discriminant 4 . Then

$$
F(x, y)=\frac{v}{w} N \Phi(x, y)
$$

where $v, w \in \boldsymbol{Z},(v, w)=1, \Phi \in \boldsymbol{K}[x, y]$ has integral eocficients and (90)

$$
\left(\Phi(x, y), \Phi^{\prime}(x, y)\right)=1,
$$

where $\Phi^{\prime}$ is conjugate to $\Phi$ over $\boldsymbol{Q}(x, y)$. Let

$$
\frac{w}{v} f_{0}(\mu \boldsymbol{t}+\tau)=\eta_{i}(\boldsymbol{t}) \eta_{i}^{\prime}(\boldsymbol{t}) \quad(i \leqslant I)
$$

be all the factorizations of the left-hand. side into two conjugate polynomials with integral coefficients in $\boldsymbol{K}$. Since $\boldsymbol{K}$ has finitely many units the number of such factorizations is finite. Fl implies that if $\left(m, \Delta f_{0}\left(\mu \boldsymbol{t}_{\mathbf{1}}+\boldsymbol{r}\right)\right)$
$=1$ there exist an $i \leqslant I$, a $\boldsymbol{t}_{2} \equiv \boldsymbol{t}_{1} \bmod m$ and $x, y \in \boldsymbol{Z}$ such that

$$
\Phi(x, y)=\eta_{i}\left(\boldsymbol{t}_{2}\right)
$$

Indeed, by (84) and (85) we have

$$
\left(\frac{w}{v} f_{0}(\mu \boldsymbol{t}+\tau)\right)=\left(\frac{w}{v} B\right) \prod_{\nu=1}^{n} \frac{\left(\psi_{\nu}(t)\right)^{e_{\nu}}}{C\left(\psi_{\nu}\right)^{e_{\nu}}}
$$

Since by Lemma $5 \prod_{\nu \text { an } 1}^{n} \frac{N \psi_{v}(\boldsymbol{t})}{N O\left(\psi_{v}\right)}$ has the fixed divisor $1, \prod_{\nu=1}^{n} \psi_{\nu}(\boldsymbol{t})^{e_{v}}$ has the fixed divisor $\prod_{p=1}^{n} O\left(\psi_{\nu}\right)^{e_{p}}$. On the other hand, for every $\boldsymbol{t} \in \mathbf{Z}^{r}$

$$
\frac{w}{v} f_{0}(\mu \boldsymbol{t}+\tau)=N \Phi(x, y) \in \boldsymbol{Z}
$$

hence

$$
\frac{w}{v} A \in \boldsymbol{Z}
$$

By (84) and (85) the condition. $\left(m, \Delta f_{0}\left(\mu \boldsymbol{t}_{1}+\tau\right)\right)=1$ implies

$$
\left(m, \Delta \prod_{\nu=1}^{n} \frac{N \psi_{\nu}\left(\boldsymbol{t}_{1}\right)}{N O\left(\psi_{v}\right)}\right)=1
$$

and by Lemma $5 \mathrm{H}$ implies the existence of a $\boldsymbol{t}_{2} \equiv \boldsymbol{t}_{1} \bmod m$ such that $\frac{\left(\psi_{\nu}\left(\boldsymbol{t}_{2}\right)\right)}{O\left(\psi_{\nu}\right)}(\nu \leqslant n)$ are distinct prime ideals not dividing $w B$. By the assumption of $\mathrm{D}$ there exist $x_{0}, y_{0} \in \boldsymbol{Z}$ such that

$$
N \Phi\left(x_{0}, y_{0}\right)=\frac{w}{v} F\left(x_{0}, y_{0}\right)=\frac{w}{v} f\left(\mu t_{2}+\tau\right)
$$

and it follows from (93) and (94) that for an integral ideal $b$ and some integers $a_{v} \geqslant 0$

$$
\left\langle\Phi\left(x_{0}, y_{0}\right)\right\rangle=\mathfrak{b} \prod_{\nu=1}^{n} \frac{\left(\psi_{\nu}\left(\boldsymbol{t}_{2}\right)\right)^{\alpha_{\nu}}}{O\left(\psi_{\nu}\right)^{a_{\nu}}}, \quad\left(\mathfrak{b}, \prod_{\nu=1}^{n} \frac{\left(\psi_{\nu}\left(\boldsymbol{t}_{2}\right)\right)}{O\left(\psi_{\nu}\right)}\right)=1 .
$$

On the other hand in full analogy with (51) we ean assume that for a certain $k=n \bmod 2$

(96) $\quad \psi_{\nu}^{\prime}=\psi_{\nu^{\prime}}, \quad e_{\nu^{\prime}}=e_{\nu^{\prime}}, \quad \nu^{\prime}=\nu(\nu \leqslant k), \quad \nu^{\prime}=\nu-(-1)^{n-\nu}(\nu>k)$.

Hence

$$
N \Phi\left(x_{0}, y_{0}\right)=N \mathfrak{b} \prod_{\nu=1}^{n}\left(\frac{\psi_{p}\left(t_{2}\right)}{O\left(\psi_{v}\right)}\right)^{\alpha_{p}+a_{p^{\prime}}}, \quad\left(N \mathrm{~b}, \prod_{\nu=1}^{n} \frac{\left(\psi_{\nu}\left(\boldsymbol{t}_{2}\right)\right)}{O\left(\psi_{\nu}\right)}\right)=1
$$


and a comparison with (93) gives

(97)

$$
a_{\nu}+a_{\nu^{\prime}}=e_{\nu} \quad(1 \leqslant \nu \leqslant n) .
$$

Now let us put

$$
\eta(\boldsymbol{t})=\Phi\left(x_{0}, y_{0}\right) \prod_{\nu=1}^{n}\left(\frac{\psi_{\nu}(\boldsymbol{t})}{\psi_{\nu}\left(\boldsymbol{t}_{2}\right)}\right)^{a_{\nu}}
$$

The polynomial $\eta(\boldsymbol{t})$ has integral coefficients in $\boldsymbol{K}$ since

$$
O(\eta)=\left\langle\Phi\left(x_{0}, y_{0}\right)\right\rangle \prod_{v=1}^{n} \frac{O\left(\psi_{v}\right)^{a_{v}}}{\left(\psi_{n}\left(\boldsymbol{t}_{2}\right)\right)^{a_{v}}}=\mathfrak{b}
$$

Moreover, by (95), (96), (97) and (84)

$$
\begin{aligned}
\eta(\boldsymbol{t}) \eta^{\prime}(\boldsymbol{t}) & =N \Phi\left(x_{0}, y_{0}\right) \prod_{\nu=1}^{n}\left(\frac{\psi_{v}(\boldsymbol{t}) \psi_{v}^{\prime}(\boldsymbol{t})}{\psi_{v}\left(\boldsymbol{t}_{2}\right) \psi_{v}^{\prime}\left(\boldsymbol{t}_{2}\right)}\right)^{\alpha_{\nu}}=\frac{w}{v} f_{0}\left(\mu \boldsymbol{t}_{2}+\tau\right) \prod_{\nu=1}^{n}\left(\frac{\psi_{v}(\boldsymbol{t})}{\psi_{\nu}\left(\boldsymbol{t}_{2}\right)}\right)^{a+a_{\nu}} \\
& =\frac{w}{v} f_{0}\left(\mu \boldsymbol{t}_{2}+\tau\right) \prod_{\nu=1}^{n}\left(\frac{\psi_{v}(\boldsymbol{t})}{\psi_{\nu}\left(\boldsymbol{t}_{2}\right)}\right)^{e_{v}}=\frac{w}{v} f_{0}(\mu \boldsymbol{t}+\tau) .
\end{aligned}
$$

Hence $\eta(t)=\eta_{i}(\boldsymbol{t})$ for an $i \leqslant I$ and (92) follows immediately from (98). Now we apply Lemma 11 with $p(\boldsymbol{t})=\Delta f_{0}(\mu \boldsymbol{t}+\boldsymbol{\tau})$,

$$
G(x, y)=\Phi(x, y)+\Phi^{\prime}(x, y), \quad H(x, y)=\left(\Phi(x, y)-\Phi^{\prime}(x, y)\right) / \sqrt{\Lambda}
$$

and we get the existence of $X_{0}, X_{0} \in Q[\boldsymbol{t}]$ satisfying

$$
\Phi\left(X_{0}, Y_{0}\right)=\eta_{j}, \quad \Phi^{\prime}\left(X_{0}, X_{0}\right)=\eta_{j}^{\prime}
$$

for a $j \leqslant I$. Using again the transformation (88) we get by (89) and (90)

$$
F(\boldsymbol{X}(\boldsymbol{t}), \mathbf{Y}(\boldsymbol{t}))=\frac{v}{w} \eta_{j}\left(\frac{\boldsymbol{t}-e \tau-\boldsymbol{t}_{0}}{e \mu}\right) \eta_{j}^{\prime}\left(\frac{\boldsymbol{t}-e \tau-\boldsymbol{t}_{0}}{e \mu}\right)=\frac{v}{w} f_{0}\left(\frac{\boldsymbol{t}-\boldsymbol{t}_{0}}{e}\right)=f(\boldsymbol{t}) .
$$

Lemma 12. Let $k \in \boldsymbol{N}$ be odd $, a_{i}(\boldsymbol{t}) \in \boldsymbol{Z}[\boldsymbol{t}]\left(0 \leqslant i \leqslant \gamma_{i}\right), a_{0}(\boldsymbol{t})=1, x(\boldsymbol{t})$ $\in \boldsymbol{Q}[\boldsymbol{t}] . I f$

$(100)$

then $x(\boldsymbol{t}) \in \boldsymbol{Z}[\boldsymbol{t}]$.

$$
\sum_{i=0}^{k-1}\left(\begin{array}{c}
k \\
i+1
\end{array}\right) a_{i}(\boldsymbol{t}) x(\boldsymbol{t})^{h-1-i}=0
$$

Proof. Suppose that $O(x) \notin \boldsymbol{Z}$. Then for some prime $p$

$$
\operatorname{ord}_{p} O(x)=-c \leqslant-1 \text {. }
$$

The function ord ${ }_{p} C\left(\mathcal{P}^{P}\right)$ is a valuation of the ring $\boldsymbol{Q}[\boldsymbol{t}]$ (soe $[6]$, p. 171). In virtue of the properties of valuations (100) implies

$$
\operatorname{ord}_{p}\left(70 C(x)^{k-1}\right) \geqslant \min _{0<i<i c} \operatorname{ord}_{p}\left(\left(\begin{array}{c}
k \\
i+1
\end{array}\right) O\left(a_{i}\right) O(x)^{k-1-i}\right)
$$

hence for a positive $i<7$

$$
\operatorname{ord}_{p} k-(k-1) e \geqslant \operatorname{ord}_{p}\left(\begin{array}{c}
k \\
i+1
\end{array}\right)-(k-1-i) e
$$

and

(10.1)

However

$$
\operatorname{ord}_{p} p \geqslant \operatorname{ord}_{p}\left(\begin{array}{c}
l \\
i+1
\end{array}\right)+i
$$

$$
\left(\begin{array}{c}
7 b \\
i+1
\end{array}\right)=\frac{k}{i+1}\left(\begin{array}{c}
k-1 \\
i
\end{array}\right)
$$

thus (101) implies

$$
\operatorname{ord}_{p}(i+1) \geqslant i, \quad i+1 \geqslant p^{i} ; \quad p=2,
$$

which is impossible since then the left-hand side of (101) is 0 .

Proof of Theorem 4. Let $n=2^{\alpha} k, k$ odd. In order to prove the first part of the theorem let us assume that the fixed divisor of $f$ equals $O(f)$ and take in the proof of Theorem $3 f_{0}=f$. If $k>1$ we take further $\boldsymbol{K}=\boldsymbol{Q}, \mu=1, \tau=\mathbf{0}$,

$$
G(x, y)=: x^{2^{a}}+y^{2^{\alpha}}, \quad H(x, y)=\sum_{i+j=k-1} x^{2^{a^{a}}}\left(-y^{2^{\alpha}}\right)^{j}
$$

and we get from (86) and (88) that for some polynomials $g, h \in \boldsymbol{Z}[\boldsymbol{t}]$ and $X, Y \in Q[t]$

(102)

$$
g(\boldsymbol{t}) h(\boldsymbol{t})=f(\boldsymbol{t}),
$$

However

$$
H(X, X)=\sum_{i=0}^{k-1}\left(\begin{array}{c}
k \\
i+1
\end{array}\right) G(X, Y)^{i}\left(-X^{2^{a}}\right)^{k-1-i}
$$

heneo taking in Lomma 12

$$
a_{i}(\boldsymbol{t})=g(\boldsymbol{t})^{i} \quad(0 \leqslant i<k-1), \quad a_{k-1}(\boldsymbol{t})=-h(\boldsymbol{t}), \quad x(\boldsymbol{t})=-X(\boldsymbol{t})^{2^{a}}
$$

we get from (102) that

$$
-X(\boldsymbol{t})^{2^{a}} \in \boldsymbol{Z}[\boldsymbol{t}]
$$

Thus $X(t) \in Z[t]$ and by symmetry $Y(t) \in Z[t]$. Moreover

$$
X(\boldsymbol{t})^{n}+Y(\boldsymbol{t})^{n}=G(X, X) E r(X, X)=f(\boldsymbol{t}) .
$$

If $\hbar_{0}=1$ we take in the proof of theorem $3 \boldsymbol{K}=Q\left(\zeta_{4}\right)$,

$$
\text { (103) } \quad \Phi(x, y)=x^{2^{\alpha-1}}+\xi_{4} y^{2^{\alpha-1}}, \quad v / w=1,
$$

where $\zeta_{q}$ is a primitive $q$ th root of unity. 
By Lemma $5 \mu$ factorizes in $\boldsymbol{K}$ into prime ideals of degree 2. By (92) and (99) for some polynomials $\eta \in Z\left[\zeta_{4}, t\right]$ and $X_{0}, Y_{0} \in Q[\boldsymbol{t}]$

(104) $\quad \eta(\boldsymbol{t}) \eta^{\prime}(\boldsymbol{t})=f(\mu \boldsymbol{t}+\tau), \quad \eta^{\mathbf{A}}$ conjugate to $\eta$ over $\boldsymbol{Q}(\boldsymbol{t})$,

(105) $\Phi\left(X_{0}(t), Y_{0}(t)\right)=\eta(t)$.

Let us set

(106) $\vartheta(\boldsymbol{t})=\eta\left(\frac{\boldsymbol{t}-\boldsymbol{\tau}}{\mu}\right), \quad X(\boldsymbol{t})=X_{0}\left(\frac{\boldsymbol{t}-\tau}{\mu}\right), \quad \bar{Y}(\boldsymbol{t})=X_{0}\left(\frac{\boldsymbol{t}-\tau}{\mu}\right)$.

We have

$$
\mu^{|\eta|} \vartheta(\boldsymbol{t}) \in \boldsymbol{Z}\left[\zeta_{4}, \boldsymbol{t}\right]
$$

hence if $\mathfrak{p}$ is a prime ideal of $\boldsymbol{K}$ in the denominator of $C(\vartheta) \mathfrak{p} \mid \mu$ and $\mathfrak{p}=\mathfrak{p}^{\prime}$. However by (104)

$$
\vartheta(\boldsymbol{t}) \vartheta^{\prime}(\boldsymbol{t})=f(\boldsymbol{t}), \quad N C(\vartheta)=O(f) \in \boldsymbol{Z}
$$

hence $\operatorname{ord}_{\mathfrak{p}} C(\vartheta)=\frac{1}{2} \operatorname{ord}_{\mathrm{p}} C(f) \geqslant 0$ and

$$
\vartheta(\boldsymbol{t}) \in \boldsymbol{Z}\left[\zeta_{4}, \boldsymbol{t}\right]
$$

Now (103), (105) and (106) imply

$$
X(\boldsymbol{t})^{2^{\alpha-1}}, Y(\boldsymbol{t})^{2^{\alpha-1}} \in \boldsymbol{Z}[\boldsymbol{t}] ; \quad X(\boldsymbol{t}), X(\boldsymbol{t}) \in \boldsymbol{Z}[\boldsymbol{t}]
$$

and we get by (104)

$$
X(\boldsymbol{t})^{2^{\alpha}}+Y(\boldsymbol{t})^{2^{a}}=f(\boldsymbol{t})
$$

The proof of the first part of the theorem is complete. In order to prove the second part it is enough to consider the case $n>2$ (for $n=2$ the assertion is contained in Theorem 1).

Let $p$ be at prime satisfying

$$
p=1 \bmod 2^{a+1}, \quad p \neq 1 \bmod 2 n \quad \text { if } \quad n \neq 2^{a}
$$

and let us choose an integer $c$ such that

$$
0^{n}+1=0 \bmod p^{n}, \quad c=-1 \quad \text { if } \quad \alpha=0 .
$$

Consider now the polynomial

$$
f(t)=u(t)^{n}+v(t)^{n},
$$

where $u(t)=\frac{t(t-1) \ldots(t-p+1)}{p}, v(t)=c u(t)+p^{n-1}$.

It is easily seen that $f(t) \in \boldsymbol{Z}[t]$ and

$$
|f|=\left\{\begin{array}{lll}
p^{n} & \text { if } & \alpha>0 \\
p^{n-1} & \text { if } & \alpha=0
\end{array}\right.
$$

Moreover since polynomials $u(t), v(t)$ are integer-valued the equation $x^{n}+y^{n}=f(t)$ is soluble in $x, y \in \boldsymbol{Z}$ for all $t \in \boldsymbol{Z}$. On the other hand suppose that

$$
X(t)^{n}+X(t)^{n}=f(t), \quad X, X \in \mathbb{Z}[t] .
$$

Since

$$
X(t)^{n}+X(t)^{n}=\prod_{i=0}^{n-1}\left(X(t)-\zeta_{2 n}^{2 i+1} Y(t)\right)
$$

we have

$$
|f| \geqslant\left\{\begin{array}{lll}
n \max \{|X|,|Y|\} & \text { if } & \alpha>0 \\
(n-1) \max \{|X|,|Y|\} & \text { if } & \alpha=0
\end{array}\right.
$$

Hence by (109)

$$
\max \{|X|,|X|\} \leqslant p
$$

Taking $i=0,1, \ldots, p-1$ we get $u(i)=0$ hence

$$
X(i)^{n}+Y(i)^{n}=p^{n(n-1)} \text {. }
$$

If $n=2^{\alpha}, \alpha>1$ or $n=3$ by special cases of Fermat's last theorem (111) implies

$$
X(i) \Psi(i)=0 \quad(0 \leqslant i<p) .
$$

If $n>3$, by Zsigmondy's theorem either $X(i) \bar{X}(i)=0$ or $X(i)= \pm \bar{X}(i)$ or $X(i)^{n}+Y(i)^{n}$ has the so-called primitive prime factor $\equiv 1 \bmod 2 n$. The last two possibilities are incompatible with (107) and (112) hence (113) holds for all $n>2$. By (112) if $X(i)=0, Y(i)=p^{n-1}$ for $\alpha=0, Y(i)= \pm p^{n-1}$ for $a>0$. In view of symmetry between $X$ and $Y$ we may assume that there is a set $S \subset\{0,1, \ldots, p-1\}$ with the following properties

$$
|S| \geqslant \frac{p+1}{2(n, 2)}, \quad X(i)=0, \quad Y(i)=p^{n-1} \quad \text { for } i \in S .
$$

(If $n$ is oven wo can replace $X$ by $-X$.) Let

$$
P(t)=\cdots \prod_{t \in S}(t-i)
$$

It follows that

(114) $|P| \geqslant \frac{p+1}{2(n, 2)}, \quad X(t)=0 \bmod P(t), \quad Y(t)=p^{n-1} \bmod P(t)$ and we get from (108) and (110)

$$
Y(t)^{n} \equiv v(t)^{n} \bmod P(t)^{n}
$$


Since $Y(t) \equiv v(t) \bmod P(t)$ and $(v, P)=1$ we obtain

$$
Y(t) \equiv v(t) \bmod P(t)^{n} .
$$

However by (111)

$$
\max \{|Y|,|v|\} \leqslant p<n|P|
$$

hence

$$
Y(t)=v(t) \notin Z[t] .
$$

\section{References}

[1] P. T. Bateman and R. A. Forn, A heuristic asymptotio formula concerning the distribution of prime numbers, Math. Comp. 16 (1962), pp. 363-367.

[2] A. Clu âtelet, Lesons sur la théorie des nombres, Paris 1913.

[3] S. Ch ow la, Some problems of elementary mumber theory, J. Reine Angow. Math.

[4] H. Davenport, D. J. Lewis and A. Schinzel, Polynomials of certain special types, Acta Arith. 9 (1964), pp. 107-116.

[5] H. Halberstam and. H. E. Richert, Sieve methods, London-Now York-San Franciseo 1974.

[6] H. Hasse, Zahlentheorie, Berlin 1963.

[7] T. K ojima, Note on number-theoretical properties of algebraio functions, Tobloku Math. J. 8 (1915), pp. 24-87.

[8] S. Lang, Diophantine geometry, New York-London 1962

[9] W. J. LeVequo, A brief survey of diophantine equations, Studjos in number theory, pp. 4-24, Englewood Cliffs N.J. 1969.

[10] D. Mumford, A. remark on Mordell's eonjecture, Amer. J. Math. 87 (1965), pp. 1007-1016.

[11] P. Ribenboim, Polynomials whose values are powers, J. Reine Angew. Math. 268/269 (1974), pp. 34-40.

[12] A. Schinzel et W. Sierpiński, Sur certaines hypothèses concernant les numbres premiers, Acta Arith. 4 (1958), pp. 185-208, Erratum 5 (1959), p. 259.

[13] A. Schinzel, On Hilbert's irreducibility theorem, Ann. Polon. Math. 16 (1965), pp. 333-340.

[14] - On a theorem of Bauer and some of its applisations II, Acta Arith. 22 (1972), pp. $221-231$.

[15] - Abetian binomials, power residues and exponential congruenoes, ibid. 32 (1977), pp. $245-274$

[16] - Addendum and corrigendum to [15], ibid. 36 (1980), pp. 101-104.

[17] W. M. Sohmidt, Equations over finite fields. An elementary approaoh, Lucturo Notes in Mathematics No 536, Berlin-Feidelborg-Now York 1970.

[18] T. Skolom, Anwendung exponentieller Kongruenzen zum Boweis der Unlösburlwoic gewisser diophantisoher Gleichungen, Vid. alsad. Avh. Oslo I., 1937, nx 12.

\section{Corrigendum to the paper "Periodic analogues of the Euler-Maclaurin and Poisson summation formulas with applications to number theory", Acta Arith. 28 (1975), pp. 23-68}

by

BRUCA O. BERNDA (Urbana, Tll.) and LOWLLL SOHOENFELD (Buffalo, N.Y.)

There is a misprint in the formulation of Proposition 9.1 on p. 55 The correct formulation is as follows:

PROPOSIntoN 9.1. For $|y|<2 \pi / k$,

$$
\frac{y \sum_{n=0}^{k \cdots 1} a_{n} e^{n y}}{e^{k y}-1}=\sum_{j=0}^{\infty} \frac{B_{j}(A)}{j !} y^{j}=e^{B(-A) y},
$$

where the last expression uses the umbral convention according to which after the formal expansion into power series, the expression $\{B(A)\}^{j}$ is to be replaced by $B_{j}(A)$.

Moreover on p. 29 , line 3 replace $1 \leqslant m \leqslant r$ by $2 \leqslant m \leqslant r$ and on $\mathrm{D}$. 30, line 10 replace $P$ by $P_{j}$.

Received on 14.7 .1980 